\title{
Resistant sets in the unit hypercube
}

\author{
Ahmad Abdi \\ Gérard Cornuéjols \\ Dabeen Lee
}

October 28, 2019

\begin{abstract}
Ideal matrices and clutters are prevalent in Combinatorial Optimization, ranging from balanced matrices, clutters of $T$-joins, to clutters of rooted arborescences. Most of the known examples of ideal clutters are combinatorial in nature. In this paper, rendered by the recently developed theory of cuboids, we provide a different class of ideal clutters, one that is geometric in nature. The advantage of this new class of ideal clutters is that it allows for infinitely many ideal minimally non-packing clutters. We characterize the densest ideal minimally non-packing clutters of the class. Using the tools developed, we then verify the Replication Conjecture for the class.
\end{abstract}

\section{Introduction}

Let $E$ be a finite set of elements, and let $\mathcal{C}$ be a family of subsets of $E$ called members. If no member is contained in another one, then $\mathcal{C}$ is a clutter over ground set $E$ [15]. The incidence matrix of $\mathcal{C}$, denoted $M(\mathcal{C})$, is the matrix whose columns are labeled by $E$ and whose rows are the incidence vectors of the members. We say that $\mathcal{C}$ is ideal if the set covering polyhedron $\left\{x \in \mathbb{R}^{E}: M(\mathcal{C}) x \geq \mathbf{1}, x \geq \mathbf{0}\right\}$ is integral [13]. Ideal clutters are prevalent in the literature:

- $M(\mathcal{C})$ is totally unimodular [22] or balanced [8] (also see [11], Chapter 6),

- $\mathcal{C}$ is the clutter of $T$-joins of a graft [17] (also see [11], Theorems 1.21 and 2.1),

- $\mathcal{C}$ is the clutter of odd circuits of a signed graph without an odd- $K_{5}$ minor [19],

- $\mathcal{C}$ is the clutter of rooted arborescences of a directed graph [14, 18] (also see [5]).

All of these clutters are ideal because the members conform to a combinatorial pattern. In this paper, we come up with a different class of clutters that is ideal because a geometric pattern is followed. ${ }^{1}$ To present this new class of ideal clutters, we need to move to a different, yet equivalent framework.

\footnotetext{
${ }^{1}$ This idea first appeared in a paper by Jon Lee [24].
} 

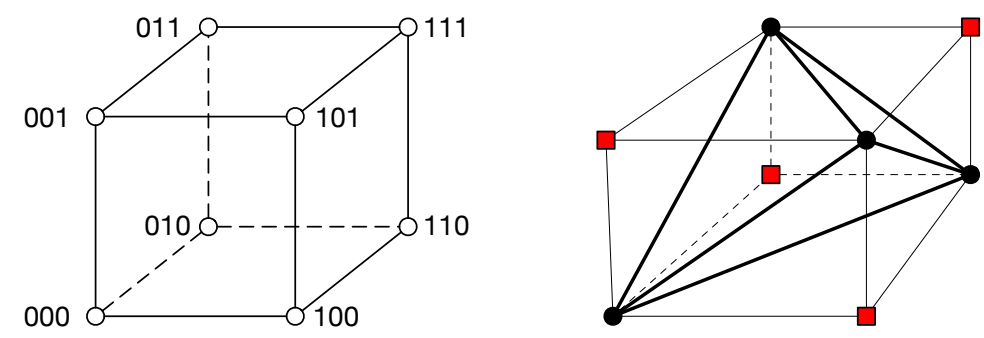

Figure 1: An illustration of the coordinate system, and the convex hull of $R_{1,1}$.

Take an integer $n \geq 1$. A cuboid is a clutter over ground set $[2 n]:=\{1, \ldots, 2 n\}$ where every member $C$ satisfies the following:

$$
|C \cap\{2 i-1,2 i\}|=1 \quad \forall i \in[n] .
$$

For instance, the clutter $Q_{6}:=\{\{2,4,6\},\{1,3,6\},\{1,4,5\},\{2,3,5\}\}$ of triangles of $K_{4}$ is a cuboid.

When is a cuboid ideal?

This question is actually equivalent to asking when a clutter is ideal, even though cuboids form a special class of clutters [3]. To answer it, we need to view cuboids as vertex subsets of the unit $n$-dimensional hypercube. To this end, denote by $\{0,1\}^{n}$ the vertices of the $n$-dimensional unit hypercube. Take a set $S \subseteq\{0,1\}^{n}$. We will think of the points in $S$ as feasible points and the points in $\bar{S}$ as infeasible points. The cuboid of $S$, denoted $\operatorname{cuboid}(S)$, is the clutter over ground set $[2 n]$ whose members have incidence vectors

$$
\left(x_{1}, 1-x_{1}, \ldots, x_{n}, 1-x_{n}\right) \quad x \in S .
$$

Notice that every cuboid over ground set $[2 n]$ is the cuboid of an appropriate subset of $\{0,1\}^{n}$. For instance, $Q_{6}$ is the cuboid of the set $R_{1,1}:=\{000,110,101,011\} \subseteq\{0,1\}^{3}$.

Theorem 1.1 ([3], Theorem 1.6). Take an integer $n \geq 1$ and a set $S \subseteq\{0,1\}^{n}$. Then cuboid $(S)$ is ideal if, and only if, $S$ is cube-ideal. ${ }^{2}$

We say that $S$ is cube-ideal if the convex hull of the feasible points is described by inequalities of the form

$$
\begin{aligned}
& 0 \leq x_{i} \leq 1 \quad i \in[n] \quad \text { (hypercube inequalities) } \\
& \sum_{i \in I} x_{i}+\sum_{j \in J}\left(1-x_{j}\right) \geq 1 \quad I, J \subseteq[n], I \cap J=\emptyset \quad \text { (generalized set covering inequalities). }
\end{aligned}
$$

For instance, the set $R_{1,1}$ is cube-ideal (see Figure 1), so by Theorem 1.1, $Q_{6}$ is an ideal cuboid.

By Theorem 1.1, our question above is equivalent to the following:

When is a set cube-ideal?

\footnotetext{
${ }^{2}$ The dual of this statement was proved in [20,27], where idealness of $0, \pm 1$ matrices was reduced to idealness of 0,1 matrices.
} 
To answer it, take a coordinate $i \in[n]$. Denote by $e_{i}$ the $i^{\text {th }}$ unit vector. To twist $S$ at coordinate $i$ is to replace $S$ by the set

$$
S \triangle e_{i}:=\left\{x \triangle e_{i}: x \in S\right\},
$$

where the second $\triangle$ refers to coordinate-wise addition modulo 2 . As twists correspond to the change of variables $x_{i} \mapsto 1-x_{i}, i \in[n]$, and the hypercube and generalized set covering inequalities are closed under these transformations, if a set is cube-ideal, then so is every twisting of it.

Given a point $x \in\{0,1\}^{n}$, the induced clutter of $S$ with respect to $x$ is the clutter over ground set $[n]$ whose members are

$$
\operatorname{ind}(S \triangle x):=\text { the minimal sets of }\left\{C \subseteq[n]: \chi_{C} \in S \triangle x\right\},
$$

where $\chi_{C} \in\{0,1\}^{n}$ is the incidence vector of $C$. Notice that if $x$ is feasible, then $\operatorname{ind}(S \triangle x)=\{\emptyset\}$. Notice that the induced clutters of $S$ pick up only local information about the set.

Theorem 1.2 ([3], Theorem 1.8). A set is cube-ideal if, and only if, every induced clutter is ideal.

This is the key to generating a new class of ideal clutters.

\subsection{Resistant sets}

Take an integer $n \geq 1$ and a set $S \subseteq\{0,1\}^{n}$. We say that $S$ is resistant if for every induced clutter, the members are pairwise disjoint. For instance, the set $R_{1,1}=\{000,110,101,011\}$ is resistant as its induced clutters are equal to either $\{\emptyset\}$ or $\{\{1\},\{2\},\{3\}\}$. Clearly, if a set is resistant, then so is every twisting of it.

Remark 1.3. A clutter whose members are pairwise disjoint is ideal.

An immediate consequence of Theorem 1.2 and Remark 1.3 is that,

Corollary 1.4. Resistant sets are cube-ideal.

Combining this with Theorem 1.1, we obtain a new class of ideal clutters:

Corollary 1.5. Cuboids of resistant sets are ideal clutters.

Resistant sets form a rich class of cube-ideal sets. We will see several basic classes of resistant sets in $\S 2$; let us display one of them here. Denote by $G_{n}$ the skeleton graph of $\{0,1\}^{n}$ whose vertices are the points in $\{0,1\}^{n}$ and two points $u, v$ are adjacent if they differ in exactly one coordinate. A feasible component of $S$ is a (connected) component of the vertex induced subgraph $G_{n}[S]$, while an infeasible component of $S$ is a component of $G_{n}[\bar{S}]$. We will prove the following in $\S 2$ :

Theorem 1.6. Take an integer $n \geq 1$ and a set $S \subseteq\{0,1\}^{n}$, where every infeasible component is a hypercube or has maximum degree at most two. Then $S$ is resistant. 


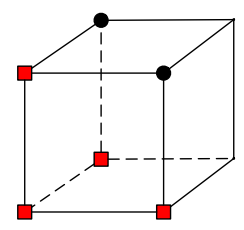

Figure 2: An illustration of a fragile set. Round points are feasible and square points are infeasible.

There are several binary operations that preserve resistance, one way or another. Take integers $n_{1}, n_{2} \geq 1$ and sets $S_{1} \subseteq\{0,1\}^{n_{1}}, S_{2} \subseteq\{0,1\}^{n_{2}}$. Define the product, coproduct and reflective product of $S_{1}, S_{2}$ as

$$
\begin{aligned}
& S_{1} \times S_{2}:=\left\{(x, y) \in\{0,1\}^{n_{1}} \times\{0,1\}^{n_{2}}: x \in S_{1} \text { and } y \in S_{2}\right\} \\
& S_{1} \oplus S_{2}:=\left\{(x, y) \in\{0,1\}^{n_{1}} \times\{0,1\}^{n_{2}}: x \in S_{1} \text { or } y \in S_{2}\right\} \\
& S_{1} * S_{2}:=\left(S_{1} \times S_{2}\right) \cup\left(\overline{S_{1}} \times \overline{S_{2}}\right),
\end{aligned}
$$

respectively. Notice that $\overline{S_{1} \oplus S_{2}}=\overline{S_{1}} \times \overline{S_{2}}$ and $\overline{S_{1} * S_{2}}=\overline{S_{1}} * S_{2}$. The following theorem is proved in $\S 2$ :

Theorem 1.7. Take integers $n_{1}, n_{2} \geq 1$ and sets $S_{1} \subseteq\{0,1\}^{n_{1}}, S_{2} \subseteq\{0,1\}^{n_{2}}$. Then the following statements hold:

(1) If $S_{1}$ is resistant, then so is $S_{1} \times\{0,1\}^{n_{2}}$.

(2) If $S_{1}, S_{2}$ are resistant, then so is $S_{1} \oplus S_{2}$.

(3) If $S_{1}, \overline{S_{1}}, S_{2}, \overline{S_{2}}$ are resistant, then so are $S_{1} * S_{2}, \overline{S_{1} * S_{2}}$.

Take a coordinate $i \in[n]$. The set obtained from $S \cap\left\{x: x_{i}=0\right\}$ after dropping coordinate $i$ is called the 0 -restriction of $S$ at coordinate $i$, and the set obtained from $S \cap\left\{x: x_{i}=1\right\}$ after dropping coordinate $i$ is called the 1-restriction of $S$ at coordinate $i$. A restriction of $S$ is a set obtained after a series of 0 - and 1-restrictions. The projection of $S$ at coordinate $i$ is the set obtained from $S$ after dropping coordinate $i$. A projection of $S$ is a set obtained after a series of single projections. A minor of $S$ is what is obtained after a series of restrictions and projections. A minor is proper if at least one operation is applied.

We will see in $\S 3$ that if a set is resistant, then so is every minor of it. So what are the excluded minors defining resistance? We say that two sets $S, S^{\prime}$ are isomorphic, and denote it by $S \cong S^{\prime}$, if one is obtained from the other after twisting and relabeling some coordinates. Take a set $F \subseteq\{0,1\}^{3}$ such that

$$
F \cap\{000,100,010,001,101,011\}=\{101,011\} .
$$

We refer to $F$, and any set isomorphic to it, as fragile (see Figure 2). Observe that $F$ is not resistant because its induced clutter with respect to the origin has intersecting members $\{1,3\},\{2,3\}$. We will prove the following characterization of resistant sets in $\S 3$ :

Theorem 1.8. Take an integer $n \geq 1$ and a set $S \subseteq\{0,1\}^{n}$. Then the following statements are equivalent: 
(i) $S$ is resistant,

(ii) S has no fragile restriction and no $\left\{\mathbf{0}^{k}, \mathbf{1}^{k}-e_{1}\right\}, k \geq 4$ isomorphic restriction, ${ }^{3}$

(iii) S has no fragile minor.

We will prove this in $\S 3\left(\mathbf{0}^{k}, \mathbf{1}^{k}\right.$ denote the $k$-dimensional vectors whose entries are 0,1 , respectively). In that section, we will also prove the following statement:

Theorem 1.9. Take an integer $n \geq 1$ and a set $S \subseteq\{0,1\}^{n}$. Then in time $O\left(n^{4}|S|^{3}\right)$, one can test whether or not $S$ is resistant.

\subsection{When do cuboids of resistant sets have the packing property?}

Let $\mathcal{C}$ be a clutter over ground set $E$. We say that $\mathcal{C}$ packs if the maximum number of pairwise disjoint members is equal to the minimum number of elements needed to intersect every member. For instance, the clutter of edges of a bipartite graph over the vertex set packs [23], while the clutter $Q_{6}$ does not [26, 28]. Given disjoint subsets $I, J \subseteq E$, the minor of $\mathcal{C}$ obtained after deleting $I$ and contracting $J$ is the clutter over ground set $E-(I \cup J)$ whose members are

$$
\mathcal{C} \backslash I / J:=\text { the minimal sets of }\{C-J: C \in \mathcal{C}, C \cap I=\emptyset\} .
$$

A minor is proper if $I \cup J \neq \emptyset$. We say that $\mathcal{C}$ has the packing property if every minor of it, including $\mathcal{C}$ itself, packs [12]. A consequence of Lehman's theorem [25] is that clutters with the packing property are ideal [12]. The converse however is not true, even for cuboids of resistant sets, as $Q_{6}$ shows. So when do cuboids of resistant sets have the packing property?

Take an integer $n \geq 1$. Two points $a, b \in\{0,1\}^{n}$ are antipodal if $a+b=1$. We say that $S \subseteq\{0,1\}^{n}$ is polar if either there are antipodal feasible points or the feasible points all agree on a coordinate; otherwise it is non-polar. We say that $S$ is strictly polar if every restriction of it, including $S$ itself, is polar. We need the following result:

Theorem 1.10 ([3], Theorem 1.11). Take an integer $n \geq 1$ and a set $S \subseteq\{0,1\}^{n}$. Then $\operatorname{cuboid}(S)$ has the packing property if, and only if, $S$ is strictly polar and every induced clutter of it has the packing property.

The reader can easily verify the following remark:

Remark 1.11. A clutter whose members are pairwise disjoint has the packing property.

As a consequence,

Corollary 1.12. Take an integer $n \geq 1$ and a resistant set $S \subseteq\{0,1\}^{n}$. Then cuboid $(S)$ has the packing property if, and only if, $S$ is strictly polar.

In fact, we will prove the following non-trivial generalization:

\footnotetext{
${ }^{3}$ Hereinafter, the adjective "isomorphic" will be omitted from "isomorphic restriction" and "isomorphic minor".
} 
Theorem 1.13. If a set is resistant and strictly polar, then its cuboid has the max-flow min-cut property.

The definition of the max-flow min-cut property, along with the proof of Theorem 1.13, can be found in $\S 7$. This theorem verifies the Replication Conjecture of Conforti and Cornuéjols [9] for cuboids of resistant sets.

So, when is a resistant set strictly polar? We will prove in $\S 4$ the following statement:

Theorem 1.14. Take an integer $n \geq 1$ and a set $S \subseteq\{0,1\}^{n}$. Then the following statements are equivalent: (i) $S$ is resistant and strictly polar, (ii) in every restriction of $S$, either there are antipodal feasible points or the feasible points form a hypercube.

We say that $S$ is strictly non-polar if it is non-polar, but every proper restriction is polar. Notice that a set is strictly polar if, and only if, it has no strictly non-polar restriction. (Beware, a set that is not strictly polar is not necessarily strictly non-polar.) We will prove in $\S 4$ that,

Theorem 1.15. Take an integer $n \geq 1$ and a resistant set $S \subseteq\{0,1\}^{n}$. Then $S$ is strictly non-polar if, and only if, cuboid $(S)$ is an ideal minimally non-packing clutter.

A clutter is minimally non-packing if it does not pack, but every proper minor does. This theorem motivates us even further to pose the following question:

Question 1.16. What are the strictly non-polar sets that are resistant?

Even though we are not able to answer this question, we can characterize the resistant strictly non-polar sets of maximum possible cardinality. To elaborate, take an integer $n \geq 1$ and a set $S \subseteq\{0,1\}^{n}$. Observe that if $S$ is non-polar, then $|S| \leq 2^{n-1}$, and if equality holds, we say that $S$ is half-dense. In [3], strictly non-polar sets are extensively studied, and among the many identified/generated examples, a significant portion are half-dense (out of the 745 strictly non-polar sets of dimension at most 7 enumerated, 74 are half-dense). Out of their examples, an infinite class and a sporadic instance played a significant role, namely the half-dense strictly non-polar sets

$$
R_{k, 1}:=\left\{\mathbf{0}^{k+1}, \mathbf{1}^{k+1}\right\} *\{0\} \quad(k \geq 1) \quad \text { and } \quad R_{5}:=R *\{0\}
$$

where

$$
R=\left\{\sum_{i=1}^{d} e_{i}, \mathbf{1}^{4}-\sum_{i=1}^{d} e_{i}: d \in[4]\right\} .
$$

We will see in $\S 2$ that $\left\{R_{k, 1}: k \geq 1\right\} \cup\left\{R_{5}\right\}$ are also resistant sets (see Figure 3). As a first step towards answering Question 1.16, we prove the following, which is the main result of this paper:

Theorem 1.17. $\left\{R_{k, 1}: k \geq 1\right\} \cup\left\{R_{5}\right\}$ are, up to isomorphism, the only half-dense strictly non-polar sets that are resistant.

This theorem answers Question 7.4 of [3] affirmatively for cuboids of resistant sets. More precisely, as an application of Theorem 1.17, we prove the following: 


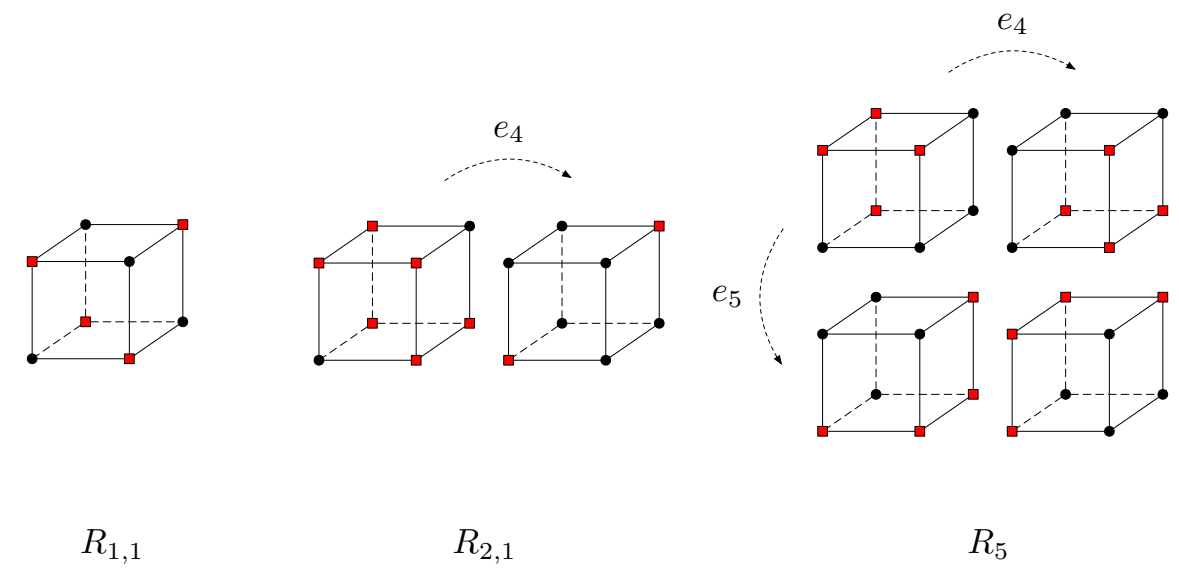

Figure 3: An illustration of $R_{1,1}, R_{2,1}, R_{5}$.

Theorem 1.18. Take integers $n_{1}, n_{2} \geq 1$ and sets $S_{1} \subseteq\{0,1\}^{n_{1}}, S_{2} \subseteq\{0,1\}^{n_{2}}$, where $S_{1}, \overline{S_{1}}, S_{2}, \overline{S_{2}}$ are nonempty and resistant. Then $S_{1} * S_{2}$ is strictly polar if, and only if, $S_{1} * S_{2}$ has none of $\left\{R_{k, 1}: k \geq 1\right\} \cup\left\{R_{5}\right\}$ as a restriction.

The proofs of these theorems can be found in $\S 6$, and the tools needed to prove them are provided in $\S 4$ and $\S 5$.

\section{Basic resistant classes and resistance-preserving operations}

In this section, we exhibit three basic classes of resistant sets as well as three operations that preserve resistance, prove Theorems 1.6 and 1.7, and show as a consequence that $\left\{R_{k, 1}: k \geq 1\right\} \cup\left\{R_{5}\right\}$ are resistant sets.

Take an integer $n \geq 1$ and a set $S \subseteq\{0,1\}^{n}$. A minimal point of $S$ is simply a point in $S$ of minimal support. We will need the following observation:

Proposition 2.1. Take an integer $n \geq 1$, a set $S \subseteq\{0,1\}^{n}$, and an infeasible component $K \subseteq \bar{S}$. Then for each $x \in K, \operatorname{ind}(S \triangle x)=\operatorname{ind}(\bar{K} \triangle x)$.

Proof. After a twisting, if necessary, we may assume that $x=\mathbf{0}$. Then we need to show that $S$ and $\bar{K}$ have the same set of minimal points. Notice that $S \subseteq \bar{K}$.

Claim 1. If $y$ is a minimal point of $\bar{K}$, then $y \in S$. In particular, the minimal points of $\bar{K}$ are also minimal points of $S$.

Proof of Claim. Since $y$ is a minimal point of $\bar{K}$, there is a hypercube $H \subseteq\{0,1\}^{n}$ such that $\mathbf{0} \in H$ and $H \cap \bar{K}=\{y\}$. In particular, $H-\{y\} \subseteq K$, implying in turn that $y$ is adjacent to a point of $K$. Since $K$ is an infeasible component of $S$ and $y \notin K$, it follows that $y \in S$.

Claim 2. The minimal points of $S$ are also minimal points of $\bar{K}$. 
Proof of Claim. Let $y$ be a minimal point of $S$. Then there is a hypercube $H \subseteq\{0,1\}^{n}$ such that $\mathbf{0} \in H$ and $H \cap S=\{y\}$. Thus, as $G_{n}[H-\{y\}]$ is connected and contains $\mathbf{0}$, it follows that $H-\{y\} \subseteq K$. As a result, $H \cap \bar{K}=\{y\}$, implying in turn that $y$ is a minimal point of $\bar{K}$, as required.

Claims 1 and 2 finish the proof.

As a consequence, the infeasible components of a set dictate whether or not a set is resistant:

Corollary 2.2. Take an integer $n \geq 1$ and a set $S \subseteq\{0,1\}^{n}$. Then the following statements are equivalent:

(i) $S$ is resistant,

(ii) for every infeasible component $K$, the set $\bar{K}$ is resistant. ${ }^{4}$

We will need the following proposition as well:

Proposition 2.3. Take an integer $n \geq 1$ and a set $S \subseteq\{0,1\}^{n}$. Then the following statements hold:

(1) If the infeasible points form a hypercube, then $S$ is resistant.

(2) If every infeasible point has at most two infeasible neighbors, then $S$ is resistant.

Proof. (1) As the infeasible points form a hypercube, $S$ does not have a 2-dimensional restriction with exactly one feasible point. Thus, a hypercube of dimension at least 2 cannot have exactly one feasible point. In particular, for each infeasible point $x$, a minimal point of $S \triangle x$ cannot have support of cardinality at least 2 . This implies that for each infeasible point $x$, the members of ind $(S \triangle x)$ have cardinality one, implying in particular that the members of ind $(S \triangle x)$ are pairwise disjoint. Therefore, $S$ is resistant. (2) Take an infeasible point $x$. We need to show that the members of ind $(S \triangle x)$ are pairwise disjoint. After a possible twisting, we may assume that $x=\mathbf{0}$, and as $x$ has at most two infeasible neighbors, we may assume after a possible relabeling that $e_{3}, e_{4}, \ldots, e_{n} \in S$. As a result, $\operatorname{ind}(S \triangle x)=\operatorname{ind}(S)$ has $\{3\},\{4\}, \ldots,\{n\}$ as members. Hence, as its ground set is $[n]$, $\operatorname{ind}(S)$ cannot have intersecting members, as required.

As a consequence,

Proof of Theorem 1.6. Take an integer $n \geq 1$ and a set $S \subseteq\{0,1\}^{n}$, where every infeasible component is a hypercube or has maximum degree at most two. It then follows from Corollary 2.2 and Proposition 2.3 that $S$ is resistant, as required.

There are also resistant sets that are the union of an arbitrary number of hypercubes that are pairwise arbitrarily far apart:

Proposition 2.4. The following statements hold:

\footnotetext{
${ }^{4}$ Notice that Proposition 2.1, together with Theorem 1.2, gives us an analogue of Remark 2.2 for cube-idealness: " $S$ is cube-ideal if, and only if, $\bar{K}$ is cube-ideal for every infeasible component $K$." This fact was noticed earlier in a much more general setting by Angulo, Ahmed, Dey, Kaibel [7], and their insight, believe it or not, had a large impact on this paper and our other papers $[3,4,6]$.
} 
(1) Take an integer $n \geq 1$ and a set $S \subseteq\{0,1\}^{n}$ that is the disjoint union of two nonempty hypercubes and contains antipodal feasible points. Then $S$ is resistant.

(2) Take an integer $p \geq 3$ and another integer $n \geq\left(\begin{array}{l}p \\ 2\end{array}\right)$. Let $\left(P_{i j}: i, j \in[p], j>i\right)$ be a partition of $[n]$ into $\left(\begin{array}{l}p \\ 2\end{array}\right)$ nonempty parts. For each $i \in[p]$, let $H_{i}$ be the hypercube of points $y \in\{0,1\}^{n}$ such that

$$
y_{k}=0 \quad \forall k \in \bigcup\left(P_{i j}: j>i\right) \text { and } y_{k}=1 \quad \forall k \in \bigcup\left(P_{j i}: j<i\right) .
$$

Then $H_{1} \cup H_{2} \cup \cdots \cup H_{p}$ is resistant.

Proof. (1) Suppose that $S$ is the disjoint union of two nonempty hypercubes $H_{1}$ and $H_{2}$, and $S$ contains antipodal points $a, b$. Since $H_{1}, H_{2}$ are nonempty and disjoint, neither of them can contain both $a$ and $b$, so we may assume that $a \in H_{1}$ and $b \in H_{2}$. Take an infeasible point $x$, if any. We need to show that the members of $\operatorname{ind}(S \triangle x)$ are pairwise disjoint. Since hypercubes (resp. antipodal points) remain hypercubes (resp. antipodal) after twists, we may assume that $x=\mathbf{0}$. It therefore suffices to show that the minimal points of $S$ have disjoint supports. As $H_{1}$ (resp. $H_{2}$ ) is a nonempty hypercube, it has a unique point of minimal support, say $a^{\prime}$ (resp. $b^{\prime}$ ). Notice that $\left\{a^{\prime}, b^{\prime}\right\}$ are the minimal points of $S$. As $a^{\prime} \leq a$ and $b^{\prime} \leq b$, and as $a, b$ are antipodal, it follows that $a^{\prime}, b^{\prime}$ have disjoint supports. Thus, $S$ is resistant.

(2) Take an infeasible point $x$. For each $i \in[p]$, let $x^{i}$ be the point in the hypercube $H_{i} \triangle x$ of minimal support. It suffices to show that $x^{1}, \ldots, x^{p}$ have pairwise disjoint supports. To this end, pick a coordinate $k \in[n]$. Choose $i, j \in[p]$ such that $j>i$ and $k \in P_{i j}$. Then $x_{k}^{i}+x_{k}^{j}=1$ and $x_{k}^{\ell}=0$ for all $\ell \in[p]-\{i, j\}$. As a result, exactly one of $x^{1}, \ldots, x^{p}$ has coordinate $k$ in its support, and since this is true for every $k \in[n]$, it follows that $x^{1}, \ldots, x^{p}$ have pairwise disjoint supports, as required.

Let us see one last example of resistant sets; this class arises from clutters. We say that $S$ is up-monotone if for all $x, y \in\{0,1\}^{n}$ such that $x \geq y$, if $y$ is feasible then so is $x$. The up-monotone set associated with a clutter $\mathcal{C}$ over ground set $E$ is

$$
\left\{\chi_{C}: C \subseteq E, C \text { contains a member of } \mathcal{C}\right\} \subseteq\{0,1\}^{E} .
$$

An element of a clutter is free if it is not contained in any member. The following remark follows rather immediately from up-monotonicity:

Remark 2.5 ([3], Remark 4.6). Take an integer $n \geq 1$, an up-monotone set $S \subseteq\{0,1\}^{n}$, and a point $x \in$ $\{0,1\}^{n}$. Then $\operatorname{ind}(S \triangle x)$ is, after deleting free elements, equal to $\operatorname{ind}(S) /\left\{i \in[n]: x_{i}=1\right\}$.

As a result,

Proposition 2.6. Let $\mathcal{C}$ be a clutter whose members are pairwise disjoint, and let $S$ be the associated upmonotone set. Then $S$ is resistant.

Proof. Take an infeasible point $x$. Then after deleting free elements, $\operatorname{ind}(S \triangle x)$ is a contraction minor of $\operatorname{ind}(S)=\mathcal{C}$, by Remark 2.5. As the members of $\mathcal{C}$ are pairwise disjoint, the members of $\operatorname{ind}(S \triangle x)$ are also pairwise disjoint. So $S$ is resistant. 
Let us now introduce three binary operations that can be used to generate resistant sets starting from smaller ones. Let $E_{1}, E_{2}$ be disjoint finite sets, and let $\mathcal{C}_{1}, \mathcal{C}_{2}$ be clutters over ground sets $E_{1}, E_{2}$, respectively. The product and coproduct of $\mathcal{C}_{1}, \mathcal{C}_{2}$ are the clutters over ground set $E_{1} \cup E_{2}$ whose members are

$$
\begin{aligned}
& \mathcal{C}_{1} \times \mathcal{C}_{2}:=\left\{C_{1} \cup C_{2}: C_{1} \in \mathcal{C}_{1}, C_{2} \in \mathcal{C}_{2}\right\} \\
& \mathcal{C}_{1} \oplus \mathcal{C}_{2}:=\text { the minimal sets of }\left\{C: C \in \mathcal{C}_{1}\right\} \cup\left\{C: C \in \mathcal{C}_{2}\right\},
\end{aligned}
$$

respectively. We need the following result:

Proposition 2.7 ([3], Remark 5.4 and Proposition 5.6). Take integers $n_{1}, n_{2} \geq 1$ and sets $S_{1} \subseteq\{0,1\}^{n_{1}}, S_{2} \subseteq$ $\{0,1\}^{n_{2}}$. Then, viewing ind $\left(S_{1}\right)$ and $\operatorname{ind}\left(S_{2}\right)$ as clutters over disjoint ground sets, we have that

$$
\begin{aligned}
& \operatorname{ind}\left(S_{1} \times S_{2}\right)=\operatorname{ind}\left(S_{1}\right) \times \operatorname{ind}\left(S_{2}\right) \\
& \operatorname{ind}\left(S_{1} \oplus S_{2}\right)=\operatorname{ind}\left(S_{1}\right) \oplus \operatorname{ind}\left(S_{2}\right)
\end{aligned}
$$

and

$$
\operatorname{ind}\left(S_{1} * S_{2}\right)= \begin{cases}\{\emptyset\} & \text { if } \mathbf{0} \in S_{1} \text { and } \mathbf{0} \in S_{2} \\ \{\emptyset\} & \text { if } \mathbf{0} \in \overline{S_{1}} \text { and } \mathbf{0} \in \overline{S_{2}} \\ \operatorname{ind}\left(\overline{S_{1}}\right) \oplus \operatorname{ind}\left(\overline{S_{2}}\right) & \text { if } \mathbf{0} \in \overline{S_{1}} \text { and } \mathbf{0} \in \overline{S_{2}} \\ \operatorname{ind}\left(\overline{S_{1}}\right) \oplus \operatorname{ind}\left(S_{2}\right) & \text { if } \mathbf{0} \in S_{1} \text { and } \mathbf{0} \in \overline{S_{2}}\end{cases}
$$

Now let us prove Theorem 1.7, telling us how to generate resistant sets starting from smaller ones:

Proof of Theorem 1.7. Take integers $n_{1}, n_{2} \geq 1$ and sets $S_{1} \subseteq\{0,1\}^{n_{1}}, S_{2} \subseteq\{0,1\}^{n_{2}}$. (1) Assume that $S_{1}$ is resistant. We need to show that $S_{1} \times\{0,1\}^{n_{2}}$ is resistant. To this end, take a point $(x, y) \in\{0,1\}^{n_{1}} \times$ $\{0,1\}^{n_{2}}$. It suffices to show that the members of $\operatorname{ind}\left(\left(S_{1} \times\{0,1\}^{n_{2}}\right) \triangle(x, y)\right)$ are pairwise disjoint. Clearly $\left(S_{1} \times\{0,1\}^{n_{2}}\right) \triangle(x, y)=\left(S_{1} \triangle x\right) \times\{0,1\}^{n_{2}}$, so by Proposition 2.7,

$$
\operatorname{ind}\left(\left(S_{1} \times\{0,1\}^{n_{2}}\right) \triangle(x, y)\right)=\operatorname{ind}\left(S_{1} \triangle x\right) \times \operatorname{ind}\left(\{0,1\}^{n_{2}}\right) .
$$

As ind $\left(\{0,1\}^{n_{2}}\right)=\{\emptyset\}$, it follows that

$$
\operatorname{ind}\left(\left(S_{1} \times\{0,1\}^{n_{2}}\right) \triangle(x, y)\right)=\operatorname{ind}\left(S_{1} \triangle x\right)
$$

As $S_{1}$ is resistant, the members of $\operatorname{ind}\left(\left(S_{1} \times\{0,1\}^{n_{2}}\right) \triangle(x, y)\right)$ must be pairwise disjoint, as required. (2) Assume that $S_{1}, S_{2}$ are resistant. We need to show that $S_{1} \oplus S_{2}$ is resistant. To this end, take a point $(x, y) \in$ $\{0,1\}^{n_{1}} \times\{0,1\}^{n_{2}}$. By Proposition 2.7,

$$
\operatorname{ind}\left(\left(S_{1} \oplus S_{2}\right) \triangle(x, y)\right)=\operatorname{ind}\left(\left(S_{1} \triangle x\right) \oplus\left(S_{2} \triangle y\right)\right)=\operatorname{ind}\left(S_{1} \triangle x\right) \oplus \operatorname{ind}\left(S_{2} \triangle y\right) .
$$

As $S_{1}, S_{2}$ are resistant, the members of $\operatorname{ind}\left(S_{1} \triangle x\right)$, ind $\left(S_{2} \triangle y\right)$ are pairwise disjoint, implying in turn that the members of ind $\left(\left(S_{1} \oplus S_{2}\right) \triangle(x, y)\right)=\operatorname{ind}\left(S_{1} \triangle x\right) \oplus \operatorname{ind}\left(S_{2} \triangle y\right)$ are pairwise disjoint, so $S_{1} \oplus S_{2}$ is resistant. (3) Assume that $S_{1}, \overline{S_{1}}, S_{2}, \overline{S_{2}}$ are resistant. We need to show that $S_{1} * S_{2}, \overline{S_{1} * S_{2}}=\overline{S_{1}} * S_{2}$ are resistant. 
Clearly, it suffices to show that $S_{1} * S_{2}$ is resistant. Pick an arbitrary point $(x, y) \in\{0,1\}^{n_{1}} \times\{0,1\}^{n_{2}}$. Then $\left(S_{1} * S_{2}\right) \triangle(x, y)=\left(S_{1} \triangle x\right) *\left(S_{2} \triangle y\right)$, so by Proposition 2.7, $\operatorname{ind}\left(\left(S_{1} * S_{2}\right) \triangle(x, y)\right)$ is one of

$$
\{\emptyset\} \quad \text { or } \quad \operatorname{ind}\left(S_{1} \triangle x\right) \oplus \operatorname{ind}\left(\overline{S_{2}} \triangle y\right) \quad \text { or } \quad \operatorname{ind}\left(\overline{S_{1}} \triangle x\right) \oplus \operatorname{ind}\left(S_{2} \triangle y\right)
$$

(notice that $\overline{S_{2} \triangle y}=\overline{S_{2}} \triangle y$ and $\overline{S_{1} \triangle x}=\overline{S_{1}} \triangle x$ ). As $S_{1}, \overline{S_{1}}, S_{2}, \overline{S_{2}}$ are all resistant, it follows that ind $\left(S_{1} \triangle x\right)$, $\operatorname{ind}\left(\overline{S_{2}} \triangle y\right), \operatorname{ind}\left(\overline{S_{1}} \triangle x\right), \operatorname{ind}\left(S_{2} \triangle y\right)$ each have only pairwise disjoint members. As a result, the members of $\operatorname{ind}\left(\left(S_{1} * S_{2}\right) \triangle(x, y)\right)$ are pairwise disjoint. Thus, $S_{1} * S_{2}$ is resistant.

We are now ready to prove the following:

Remark 2.8. $\left\{R_{k, 1}: k \geq 1\right\} \cup\left\{R_{5}\right\}$ are resistant sets.

Proof. Take an integer $k \geq 1$. Recall that $R_{k, 1}=\left\{\mathbf{0}^{k+1}, \mathbf{1}^{k+1}\right\} *\{0\}$. By Proposition $2.4(1),\left\{\mathbf{0}^{k+1}, \mathbf{1}^{k+1}\right\}$ is resistant, and by Theorem 1.6, $\overline{\left\{\mathbf{0}^{k+1}, \mathbf{1}^{k+1}\right\}}$ is resistant. It is clear that $\{0\},\{1\}$ are resistant too. As a result, by Theorem 1.7 (3), $R_{k, 1}$ is resistant. To prove that $R_{5}$ is resistant, recall that $R_{5}=R *\{0\}$ for

$$
R=\left\{\sum_{i=1}^{d} e_{i}, \mathbf{1}^{4}-\sum_{i=1}^{d} e_{i}: d \in[4]\right\} .
$$

Notice that $R, \bar{R}$ are circuits of length 8 . As a result, Theorem 1.6 implies that both $R, \bar{R}$ are resistant. Hence, Theorem 1.7 (3) implies that $R_{5}$ is resistant.

\section{Testing resistance in polynomial time}

Here we prove Theorems 1.8 and 1.9. We need the following easy remark:

Remark 3.1 ([3], Remark 2.11(i)). Take an integer $n \geq 1$ and a set $S \subseteq\{0,1\}^{n}$. Then an induced clutter of $a$ minor of $S$ is a minor of an induced clutter of $S$.

If the members of a clutter are pairwise disjoint, then so are the members of any minor of it. This fact, combined with the preceding remark, implies the following:

Remark 3.2. If a set is resistant, then so is any minor of it.

We are now ready to prove Theorem 1.8, providing two characterizations of resistant sets:

Proof of Theorem 1.8. Take an integer $n \geq 1$ and a set $S \subseteq\{0,1\}^{n}$. We need to prove that the following statements are equivalent:

(i) $S$ is resistant,

(ii) $S$ has no fragile restriction and no $\left\{\mathbf{0}^{k}, \mathbf{1}^{k}-e_{1}\right\}, k \geq 4$ restriction,

(iii) $S$ has no fragile minor. 
(i) $\Rightarrow$ (ii): As we noted already, fragile sets are not resistant. For each integer $k \geq 4$, the set $\left\{\mathbf{0}^{k}, \mathbf{1}^{k}-e_{1}\right\}$ is not resistant either, because its induced clutter with respect to $e_{1}+e_{2}$ has intersecting members $\{1,2\}$ and $\{1,3,4, \ldots, k\}$. Remark 3.2 now tells us that (i) implies (ii).

(ii) $\Rightarrow$ (iii): Assume that $S$ has a fragile minor. It suffices to show that $S$ has either a fragile restriction or a $\left\{\mathbf{0}^{k}, \mathbf{1}^{k}-e_{1}\right\}$ restriction, for some $k \in\{4, \ldots, n\}$. We will need the following two claims:

Claim 1. Suppose $T \subseteq\{0,1\}^{4}$ has no fragile restriction and its projection at coordinate 4 is fragile. Then $T$ is a twisting of $\left\{\mathbf{0}^{4}, \mathbf{1}^{4}-e_{1}\right\}$.

Proof of Claim. For $i \in\{0,1\}$, let $T_{i} \subseteq\{0,1\}^{3}$ be the $i$-restriction of $T$ at coordinate 4 . Since the projection of $T$ at 4 is fragile, it follows that $\{000,100,010,001\} \subseteq \overline{T_{0}}$ and $\{000,100,010,001\} \subseteq \overline{T_{1}}$. Moreover, as $T_{0}$ and $T_{1}$ are not fragile, we may assume that $T_{0} \cap\{101,011\}=\{011\}$ and $T_{1} \cap\{101,011\}=\{101\}$ :
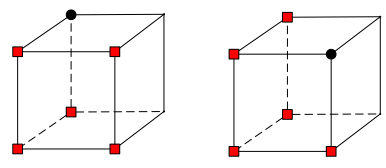

Once again, as $T_{0}$ and $T_{1}$ are not fragile, it follows that $110 \notin T_{0} \cup T_{1}$. Since the 1-restriction of $T$ at coordinate 1 is not fragile, we get that $111 \notin T_{0}$, and since the 1-restriction of $T$ at coordinate 2 is not fragile, $111 \notin T_{1}$. Thus, $T$ is a twisting of $\left\{\mathbf{0}^{4}, \mathbf{1}^{4}-e_{1}\right\}$, as claimed.

Claim 2. Take an integer $k \geq 4$ and a set $T \subseteq\{0,1\}^{k+1}$ without a $\left\{\mathbf{0}^{k}, \mathbf{1}^{k}-e_{1}\right\}$ restriction. If the projection of $T$ at coordinate $k+1$ is $\left\{\mathbf{0}^{k}, \mathbf{1}^{k}-e_{1}\right\}$, then $T$ is a twisting of $\left\{\mathbf{0}^{k+1}, \mathbf{1}^{k+1}-e_{1}\right\}$.

Proof of Claim. For $i \in\{0,1\}$, let $T_{i} \subseteq\{0,1\}^{k}$ be the $i$-restriction of $T$ at coordinate $k+1$. Clearly, $T_{i} \subseteq$ $\left\{\mathbf{0}^{k}, \mathbf{1}^{k}-e_{1}\right\}$ for each $i \in\{0,1\}$. As equality cannot hold, we may assume that $T_{0} \cap\left\{\mathbf{0}^{k}, \mathbf{1}^{k}-e_{1}\right\}=\left\{\mathbf{0}^{k}\right\}$ and $T_{1} \cap\left\{\mathbf{0}^{k}, \mathbf{1}^{k}-e_{1}\right\}=\left\{\mathbf{1}^{k}-e_{1}\right\}$, implying in turn that $T$ is a twisting of $\left\{\mathbf{0}^{k+1}, \mathbf{1}^{k+1}-e_{1}\right\}$.

Suppose a fragile minor of $S$ is obtained after applying $k$ single projections and $n-k-3$ single restrictions, for some $k \in\{0, \ldots, n-3\}$. If $k=0$, then $S$ has a fragile restriction, so we are done. We may therefore assume that $k \geq 1$ and $S$ has no fragile restriction. It follows from Claim 1 that $S$ has a $\left\{\mathbf{0}^{4}, \mathbf{1}^{4}-e_{1}\right\}$ minor obtained after applying $k-1$ single projections and $n-k-3$ single restrictions. If $k=1$, then $S$ has a $\left\{\mathbf{0}^{4}, \mathbf{1}^{4}-e_{1}\right\}$ restriction. We may therefore assume that $k \geq 2$ and $S$ has no $\left\{\mathbf{0}^{4}, \mathbf{1}^{4}-e_{1}\right\}$ restriction. Now by repeatedly applying Claim 2, we see that $S$ has one of $\left\{\mathbf{0}^{\ell}, \mathbf{1}^{\ell}-e_{1}\right\}, \ell \in\{5, \ldots, k+3\}$ as a restriction, as required.

(iii) $\Rightarrow$ (i): Assume that $S$ is not resistant. It suffices to prove that $S$ has a fragile minor. After possibly twisting $S$, we may assume that $\mathcal{C}:=\operatorname{ind}(S)$ has intersecting members.

Claim 3. There exist disjoint $I, J \subseteq[n]$ such that $\mathcal{C} \backslash I / J$ has ground set $\{x, y, z\}$ and has $\{x, z\},\{y, z\}$ among its members. 

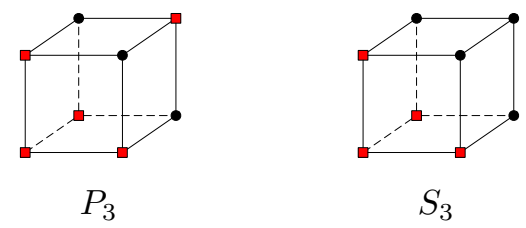

Figure 4: An illustration of $P_{3}$ and $S_{3}$, the smallest non-cube-ideal sets.

Proof of Claim. Among all pairs of intersecting members in $\mathcal{C}$, pick an intersecting pair $C_{1}, C_{2}$ whose union is minimal. Our minimal choice of $C_{1}, C_{2}$ implies that every member of $\mathcal{C}$ contained in $C_{1} \cup C_{2}$ is either $C_{1}$ or $C_{2}$ or it contains $C_{1} \triangle C_{2}$. Take elements $x \in C_{1}-C_{2}, y \in C_{2}-C_{1}$ and $z \in C_{1} \cap C_{2}$. Let $I:=[n]-\left(C_{1} \cup C_{2}\right)$ and $J:=[n]-(I \cup\{x, y, z\})$. It is easy to see that $\mathcal{C} \backslash I / J$ has ground set $\{x, y, z\}$ and has $\{x, z\},\{y, z\}$ among its members.

Consider now the minor $S^{\prime}$ of $S$ obtained after 0 -restricting coordinates $I$ and projecting away coordinates $J$. Since $\operatorname{ind}(S)=\mathcal{C}$, it follows that $\operatorname{ind}\left(S^{\prime}\right)=\mathcal{C} \backslash I / J$ has $\{x, z\},\{y, z\}$ as members, implying in turn that $S^{\prime}$ is fragile. Thus, $S$ has a fragile minor, as required.

Define $P_{3}:=\{110,011,101\} \subseteq\{0,1\}^{3}$ and $S_{3}:=\{110,011,101,111\} \subseteq\{0,1\}^{3}$ (see Figure 4). Notice that the induced clutters of $P_{3}$ and $S_{3}$ with respect to the origin are equal to $\Delta_{3}:=\{\{1,2\},\{2,3\},\{3,1\}\}$. As $\left(\frac{1}{2} \frac{1}{2} \frac{1}{2}\right)$ is an extreme point of its set covering polyhedron, $\Delta_{3}$ is non-ideal, so by Theorem $1.2, P_{3}, S_{3}$ are not cube-ideal. In fact, it can be readily checked that $P_{3}$ and $S_{3}$ are the only non-cube-ideal sets of dimension at most 3 .

Remark 3.3. Take a set $S \subseteq\{0,1\}^{3}$. Then $S$ is fragile if, and only if, $S \cup X$ is isomorphic to either $P_{3}, S_{3}$ for some set $X \subseteq\{0,1\}^{3}$ of cardinality at most one.

Thus a fragile set can be made non-cube-ideal after making at most one infeasible point feasible. Theorem 1.8 and Remark 3.3 together imply the following result, which justifies our choice of the term "resistant":

Theorem 3.4. Take an integer $n \geq 1$ and a set $S \subseteq\{0,1\}^{n}$. Then $S$ is resistant if, and only if, $S \cup X$ has no $P_{3}, S_{3}$ minor for all sets $X \subseteq\{0,1\}^{n}$ of cardinality at most one.

Proof. $(\Leftarrow)$ Assume that $S$ is not resistant. Then by Theorem 1.8 (iii), $S$ has a fragile minor $S^{\prime} \subseteq\{0,1\}^{3}$. After a possible twisting and relabeling, we may assume that $S^{\prime}$ is obtained after 0-restricting $I \subseteq[n]-\{1,2,3\}$ and projecting away $J \subseteq[n]-\{1,2,3\}$. Since $S^{\prime}$ is fragile, Remark 3.3 implies that there is a set $X^{\prime} \subseteq\{0,1\}^{3}$ of cardinality at most one such that $S^{\prime} \cup X^{\prime}$ is isomorphic to one of $P_{3}, S_{3}$. Define $X \subseteq\{0,1\}^{n}$ as follows: if $X^{\prime}=\emptyset$ set $X:=\emptyset$, otherwise set $X:=\{x\}$ where $x \in\{0,1\}^{n}$ is obtained from the point in $X^{\prime}$ by setting the coordinates in $I \cup J$ to 0 . Then $S \cup X$ has an $S^{\prime} \cup X^{\prime}$ minor obtained after 0-restricting $I$ and projecting away $J$. Since $S^{\prime} \cup X^{\prime}$ is isomorphic to one of $P_{3}, S_{3}$, we get that $S \cup X$ has one of $P_{3}, S_{3}$ as a minor, as required. ( $\Rightarrow$ ) Assume that $S \cup X$ has one of $P_{3}, S_{3}$ as minor, for some set $X \subseteq\{0,1\}^{n}$ of cardinality at most one. Then there 
is a set $Y \subseteq\{0,1\}^{3}$ of cardinality at most one such that $S$ has one of $P_{3}-Y, S_{3}-Y$ as a minor. By Remark 3.3, both $P_{3}-Y, S_{3}-Y$ are fragile, so $S$ has a fragile minor. Thus, by Theorem 1.8 (iii), $S$ is not resistant.

Take an integer $n \geq 1$ and points $a, b \in\{0,1\}^{n}$. Denote by dist $(a, b)$ the (Hamming) distance between $a$ and $b$, that is, $\operatorname{dist}(a, b)$ is the number of coordinates $a, b$ disagree on. We will next prove Theorem 1.9, stating that the resistance of $S \subseteq\{0,1\}^{n}$ can be tested in time $O\left(n^{4}|S|^{3}\right)$.

Proof of Theorem 1.9. We will take advantage of Theorem 1.8 (ii), stating that $S \subseteq\{0,1\}^{n}$ is resistant if, and only if, it has no fragile restriction and no $\left\{\mathbf{0}^{k}, \mathbf{1}^{k}-e_{1}\right\}, k \in\{4, \ldots, n\}$ restriction. For $k \in\{3,4, \ldots, n\}$, consider the following algorithm:

1. for every pair of points $x, y$ of $S$ at distance $k-1$,

(a) let $I:=\left\{i \in[n]: x_{i}=y_{i}\right\}$,

(b) for every coordinate $i \in I$,

i. let $S^{\prime} \subseteq\{0,1\}^{k}$ be the restriction of $S$ at coordinates $I-\{i\}$ containing (the images of) $x$ and $y$, that is, $S^{\prime}$ is obtained after $x_{j}$-restricting coordinate $j$ for each $j \in I-\{i\}$,

ii. if $k=3$ and $S^{\prime}$ is fragile, then output " $S$ has a fragile restriction",

iii. if $k \geq 4$ and $S^{\prime}$ is isomorphic to $\left\{\mathbf{0}^{k}, \mathbf{1}^{k}-e_{1}\right\}$, then output " $S$ has a $\left\{\mathbf{0}^{k}, \mathbf{1}^{k}-e_{1}\right\}$ restriction",

2. if $k=3$, then output " $S$ has no fragile restriction",

3. if $k \geq 4$, then output " $S$ has no $\left\{\mathbf{0}^{k}, \mathbf{1}^{k}-e_{1}\right\}$ restriction".

The correctness of this algorithm is clear; its running time is $n\left(\begin{array}{c}|S| \\ 2\end{array}\right) \times(n-k+1) \times n|S|$. Thus, by Theorem 1.8 (ii), one can test whether or not $S$ is resistant in time $\sum_{k=3}^{n} n\left(\begin{array}{c}|S| \\ 2\end{array}\right) \times(n-k+1) \times n|S|=O\left(n^{4}|S|^{3}\right)$, as required.

\section{Propagations}

In this section, we prove three lemmas needed for all the forthcoming proofs, as well as prove Theorems 1.14 and 1.15. Before getting started, let us set up a few ingredients. Recall that for an integer $n \geq 1, G_{n}$ is the skeleton graph of $\{0,1\}^{n}$.

Remark 4.1. For an integer $n \geq 1$, the following statements hold:

- For points $a, b, c \in\{0,1\}^{n}$, $\operatorname{dist}(a, b)+\operatorname{dist}(b, c) \geq \operatorname{dist}(a, c)$.

- For points $a, b \in\{0,1\}^{n}$, every $(a, b)$-path in $G_{n}$ has at least $\operatorname{dist}(a, b)$ many edges.

An $(a, b)$-path whose vertices are $a=v_{0}, v_{1}, \ldots, v_{k}=b$ as traversed from $a$ to $b$ will be represented as the sequence $\left(v_{0}, v_{1}, \ldots, v_{k}\right)$. The length of a path is the number of edges it has. An $(a, b)$-path of $G_{n}$ is straight if it has length exactly dist $(a, b)$. 
Remark 4.2. Take an integer $n \geq 1$. Then the following statements hold:

- Take distinct points $a, b$ at Hamming distance $\ell \geq 1$, and let $P$ be an $(a, b)$-path of $G_{n}$. Then $P$ is straight if, and only if, there are $\ell$ distinct coordinates $i_{1}, \ldots, i_{\ell}$ such that

$$
P=\left(a, a \triangle e_{i_{1}}, a \triangle e_{i_{1}} \triangle e_{i_{2}}, \ldots, a \triangle e_{i_{1}} \triangle e_{i_{2}} \triangle \cdots \triangle e_{i_{\ell}}=b\right) .
$$

- Pick distinct points $a, b, c$ such that $\operatorname{dist}(a, b)+\operatorname{dist}(b, c)=\operatorname{dist}(a, c)$. If $P$ is a straight $(a, b)$-path and $Q$ a straight $(b, c)$-path of $G_{n}$, then $P \cup Q$ is a straight $(a, c)$-path of $G_{n} \cdot{ }^{5}$

Take a set $S \subseteq\{0,1\}^{n}$. A path in $G_{n}[S]$ is called a feasible path, and a path in $G_{n}[\bar{S}]$ is called an infeasible path. We say that $S$ is connected if $G_{n}[S]$ is a connected graph. If every restriction of $S$, including $S$ itself, is connected, then we say that $S$ is strictly connected.

Proposition 4.3 ([3], Proposition 5.10). Take an integer $n \geq 1$ and a set $S \subseteq\{0,1\}^{n}$. Then the following statements are equivalent:

(i) $S$ is strictly connected,

(ii) S has no $\left\{\mathbf{0}^{k}, \mathbf{1}^{k}\right\}, k \geq 2$ restriction,

(iii) for all distinct feasible points $a$ and $b$, there is a straight feasible $(a, b)$-path.

\subsection{An example}

Let us start with the following proposition which best illustrates the title of this section:

Proposition 4.4. Take an integer $n \geq 1$ and a set $S \subseteq\{0,1\}^{n}$, where for all $x \in\{0,1\}^{n}$ and distinct $i, j \in[n]$, the following statement holds:

$$
\text { if } x, x \triangle e_{i}, x \triangle e_{j} \in S \text { then } x \triangle e_{i} \triangle e_{j} \in S .
$$

Then every component of $S$ is a hypercube.

Proof. Let us start with the following claim:

Claim. Let $I \subseteq[n]$ be of cardinality at least two. If $x \in S$ and $x \triangle e_{i} \in S$ for each $i \in I$, then we have $x \triangle\left(\sum_{i \in I} e_{i}\right) \in S$.

Proof of Claim. We proceed by induction on $|I| \geq 2$. The base case $|I|=2$ follows from the hypothesis of Proposition 4.4. For the induction step, assume that $k:=|I| \geq 3$. After a possible twisting and relabeling, we may assume that $x=\mathbf{0}$ and $I=\left\{e_{1}, \ldots, e_{k}\right\}$. We need to show that $\sum_{i=1}^{k} e_{i} \in S$. Let $y:=\sum_{i=1}^{k-2} e_{i}$. If $k=3$ then $y \in S$ by assumption, and if $k \geq 4$ then $y \in S$ by the induction hypothesis. Moreover, the induction hypothesis implies that $y \triangle e_{k-1}, y \triangle e_{k} \in S$. As a result, $\sum_{i=1}^{k} e_{i}=y \triangle e_{k-1} \triangle e_{k} \in S$ by the hypothesis of Proposition 4.4, thereby completing the induction step. This finishes the proof of the claim.

\footnotetext{
${ }^{5}$ If $P$ is an $(a, b)$-path and $Q$ is a $(b, c)$-path, then $P \cup Q$ denotes the $(a, c)$-walk that first traverses the vertices of $P$ from $a$ to $b$, and then traverses the vertices of $Q$ from $b$ to $c$.
} 
Take a component $S^{\prime}$ of $S$. Let $d$ be the maximum number of feasible neighbors of a point in $S^{\prime}$. If $d \leq 1$, then $\left|S^{\prime}\right| \in\{1,2\}$, so $S^{\prime}$ is clearly a hypercube. Otherwise, $d \geq 2$. After a possible twisting and relabeling, we may assume that $\mathbf{0}, e_{1}, \ldots, e_{d} \in S^{\prime}$. Then for all subsets $I \subseteq[d]$ of cardinality at least two, $\sum_{i \in I} e_{i} \in S$ by the claim. As a result,

$$
\left\{x \in\{0,1\}^{n}: x_{j}=0, j \in[n]-[d]\right\} \subseteq S^{\prime} .
$$

Since every feasible point in $S^{\prime}$ has at most $d$ feasible neighbors, equality holds above, so $S^{\prime}$ is a hypercube, as required.

So the condition "if $x, x \triangle e_{i}, x \triangle e_{j} \in S$ then $x \triangle e_{i} \triangle e_{j} \in S$ " has a propagating effect, ensuring that every feasible component is a hypercube. As a consequence,

Corollary 4.5. Take an integer $n \geq 1$ and a set $S \subseteq\{0,1\}^{n}$. Then every component of $S$ is a hypercube if, and only if, $S$ has no $\{00,10,01\}$ restriction.

Proof. $(\Rightarrow)$ If every feasible component is a hypercube, then there is no 2-dimensional restriction with exactly three feasible points, so there is no $\{00,10,01\}$ restriction. $(\Leftarrow)$ Assume that $S$ has no $\{00,10,01\}$ restriction. Then for all $x \in\{0,1\}^{n}$ and distinct $i, j \in[n]:$ if $x, x \triangle e_{i}, x \triangle e_{j} \in S$ then $x \triangle e_{i} \triangle e_{j} \in S$. Thus, by Proposition 4.4, every component of $S$ is a hypercube.

So excluding $\{00,10,01\}$ restrictions has a propagating effect. In the same vein, resistance, which is equivalent to excluding fragile restrictions and $\left\{\mathbf{0}^{k}, \mathbf{1}^{k}-e_{1}\right\}, k \geq 4$ by Theorem 1.8 (ii), entails propagations.

\subsection{Propagations in resistant sets}

Here we state three lemmas illustrating the different propagations running in resistant sets. Here is the first lemma:

Lemma 4.6 (Plane Propagation). Take an integer $n \geq 1$ and a resistant set $S \subseteq\{0,1\}^{n}$. If $S \cap\left\{x: x_{n}=\right.$ $0\}=\emptyset$, then $S$ is a hypercube.

Proof. Let $S_{1} \subseteq\{0,1\}^{n}$ be the 1-restriction of $S$ at coordinate $n$.

Claim 1. $S_{1}$ is strictly connected.

Proof of Claim. Suppose otherwise. Then by Proposition 4.3, there is an integer $k \geq 2$ such that $S_{1}$ has a $\left\{\mathbf{0}^{k}, \mathbf{1}^{k}\right\}$ restriction. Since $S \cap\left\{x: x_{n}=0\right\}=\emptyset$, it follows that $S$ has a $\left\{\mathbf{0}^{k+1}, \mathbf{1}^{k+1}-e_{1}\right\}$ restriction. As $S$ is resistant, Theorem 1.8 (ii) implies that $k=2$. However, $\left\{\mathbf{0}^{3}, \mathbf{1}^{3}-e_{1}\right\}$ is fragile, so $S$ has a fragile restriction, a contradiction to Theorem 1.8 (ii).

Claim 2. Every component of $S_{1}$ is a hypercube. 


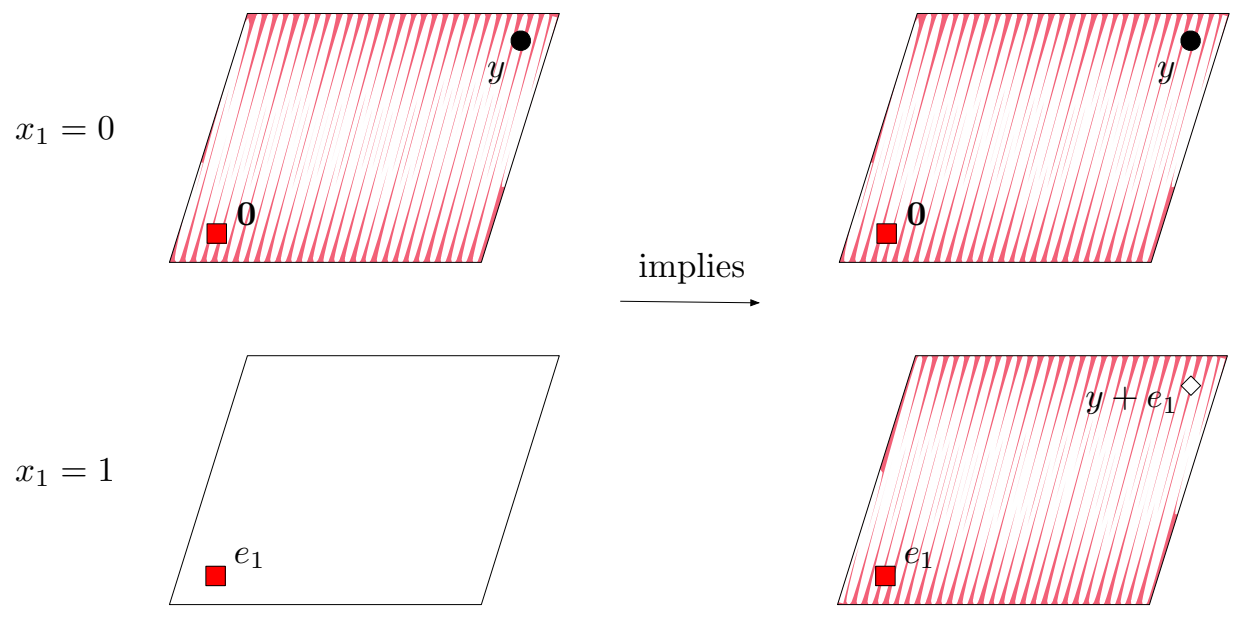

Figure 5: An illustration of Remark 4.7. Round points are feasible, the square points and the shaded region are infeasible, while the diamond point can be either.

Proof of Claim. Suppose otherwise. It follows from Corollary 4.5 that $S_{1}$ has a $\{00,10,01\}$ restriction. As $S \cap\left\{x: x_{n}=0\right\}=\emptyset$, it follows that $S$ has a $\{000,100,010\}$ restriction. However, $\{000,100,010\}$ is fragile, a contradiction to Theorem 1.8 (ii).

Claims 1 and 2 together imply that $S_{1}$ is a hypercube, so $S$ is a hypercube because $S \cap\left\{x: x_{n}=0\right\}=\emptyset$, as required.

For the next lemma, let us start with the following remark illustrated in Figure 5:

Remark 4.7. Take an integer $n \geq 1$ and a resistant set $S \subseteq\{0,1\}^{n}$, where $\mathbf{0}, e_{1}$ are infeasible. Assume that $y$ is a minimal feasible point such that $y_{1}=0$. Then $\left\{z \in S: z \leq y+e_{1}, z_{1}=1\right\} \subseteq\left\{y+e_{1}\right\}$.

Proof. Suppose otherwise. Pick a minimal point $z$ of $\left\{z \in S: z \leq y+e_{1}, z_{1}=1\right\}$. Our contrary assumption implies that $z \neq y+e_{1}$, and therefore, $z$ is also a minimal point of $S$. Moreover, as $e_{1}$ is infeasible, $z \neq e_{1}$. Pick members $C, C^{\prime} \in \operatorname{ind}(S)$ such that $y=\chi_{C}$ and $z=\chi_{C^{\prime}}$. Then $C \cap C^{\prime} \neq \emptyset$, a contradiction as $S$ is resistant.

Let us phrase this remark in a more applicable language. Take an integer $n \geq 1$ and a resistant set $S \subseteq\{0,1\}^{n}$. A valid pair is a pair $[x, y]$ where $x$ is infeasible, $y$ is feasible, and $y \triangle x$ is a minimal feasible point of $S \triangle x$. If $[x, y]$ is a valid pair, we will say that $x$ sees $y$. Remark 4.7 has the following immediate consequence:

Lemma 4.8 (Sight Propagation). Take an integer $n \geq 1$, a resistant set $S \subseteq\{0,1\}^{n}$ and a valid pair $[x, y]$. For a coordinate $i \in[n]$ such that $x \triangle e_{i}$ is infeasible, exactly one of the following statements holds:

(i) $y \triangle e_{i}$ is feasible and $\left[x \triangle e_{i}, y \triangle e_{i}\right]$ is valid,

(ii) $y \triangle e_{i}$ is infeasible and $\left[x \triangle e_{i}, y\right]$ is valid. 
Proof. After a possible twisting and relabeling, if necessary, we may assume that $x=\mathbf{0}$ and $i=1$. As $[\mathbf{0}, y]$ is valid, $y$ is a minimal feasible point. If $y_{1}=1$, then clearly (ii) holds and (i) does not. Otherwise, $y_{1}=0$. Then by Remark 4.7, $\left\{z \in S: z \leq y+e_{1}, z_{1}=1\right\}$ is either $\emptyset$ or $\left\{y+e_{1}\right\}$. In the first case, (ii) holds and (i) does not, while in the second case, (i) holds and (ii) does not.

The Sight Propagation Lemma has a subtle implication, which leads to the third propagation lemma. Take an integer $n \geq 1$, a resistant set $S \subseteq\{0,1\}^{n}$ and an infeasible point $x$. A valid sequence for $x$ is a nonempty sequence $\left(i_{1}, i_{2}, \ldots, i_{k}\right)$ of (not necessarily distinct) coordinates in $[n]$ such that the points

$$
x \triangle e_{i_{1}}, x \triangle e_{i_{1}} \triangle e_{i_{2}}, \ldots, x \triangle e_{i_{1}} \triangle e_{i_{2}} \triangle \cdots \triangle e_{i_{k}}
$$

are infeasible. Take a valid pair $[x, y]$ and a valid sequence $\left(i_{1}, \ldots, i_{k}\right)$ for $x$. In what follows, we define the trajectory of $[x, y]$ along $\left(i_{1}, \ldots, i_{k}\right)$ as some sequence $\left(t_{1}, \ldots, t_{k}\right)$ with entries in $\{0,1\}$ which will be defined precisely shortly, and given the sequence, we define the image of $[x, y]$ along $\left(i_{1}, \ldots, i_{k}\right)$ as

$$
\operatorname{im}[x, y]\left(i_{1}, \ldots, i_{k}\right):=y+\sum_{j=1}^{k} t_{j} e_{i_{j}} \quad \bmod 2 .
$$

The sequence $\left(t_{1}, \ldots, t_{k}\right)$ is defined as follows:

- for a valid pair $[x, y]$ and a valid sequence $(i)$ of length 1 , the trajectory of $[x, y]$ along $(i)$ is

$$
T[x, y](i):= \begin{cases}(1) & \text { if } y \triangle e_{i} \in S \\ (0) & \text { if } y \triangle e_{i} \notin S\end{cases}
$$

- for a valid pair $[x, y]$ and a valid sequence $\left(i_{1}, \ldots, i_{k}\right)$ of length at least 2 , the trajectory of $[x, y]$ along $\left(i_{1}, \ldots, i_{k}\right)$ is defined recursively as follows: let $y^{\prime}:=\operatorname{im}[x, y]\left(i_{1}, \ldots, i_{k-1}\right)$ and

$$
T[x, y]\left(i_{1}, \ldots, i_{k}\right):= \begin{cases}T[x, y]\left(i_{1}, \ldots, i_{k-1}\right) \cup(1) & \text { if } y^{\prime} \triangle e_{i_{k}} \in S \\ T[x, y]\left(i_{1}, \ldots, i_{k-1}\right) \cup(0) & \text { if } y^{\prime} \triangle e_{i_{k}} \notin S\end{cases}
$$

(Given two sequences $(a, \ldots, b)$ and $(c, \ldots, d),(a, \ldots, b) \cup(c, \ldots, d)$ is the sequence $(a, \ldots, b, c, \ldots, d)$.) The following is an immediate consequence of the Sight Propagation Lemma:

Remark 4.9. Take an integer $n \geq 1$, a resistant set $S \subseteq\{0,1\}^{n}$, a valid pair $[x, y]$ and a valid sequence $\left(i_{1}, \ldots, i_{k}\right)$ for $x$. Then $\operatorname{im}[x, y]\left(i_{1}, \ldots, i_{k}\right)$ is feasible and is seen by $x \Delta e_{i_{1}} \triangle \cdots \Delta e_{i_{k}}$.

We are now ready for the third propagation lemma:

Lemma 4.10 (Path Propagation). Take an integer $n \geq 1$, a resistant set $S \subseteq\{0,1\}^{n}$, a straight infeasible path $P$ contained in $\left\{x: x_{n}=0\right\}$, and let $a, b$ be the ends of $P$. If $a \triangle e_{n}, b \triangle e_{n}$ are feasible, then for every vertex $v$ of $P, v \triangle e_{n}$ is feasible. 
Proof. If $a, b$ are the only vertices of $P$, then we are clearly done. Otherwise, as $P$ is straight and contained in $\left\{x: x_{n}=0\right\}$, we may assume by Remark 4.2 that after a possible relabeling,

$$
P=\left(a=\mathbf{0}, e_{1}, e_{1}+e_{2}, \ldots, \sum_{i=1}^{k} e_{i}=b\right),
$$

where $k \in\{2, \ldots, n-1\}$. Assuming that $a \triangle e_{n}=e_{n}$ and $b \triangle e_{n}=e_{n}+\sum_{i=1}^{k} e_{i}$ are feasible, we need to show that the points $e_{n}+\sum_{i=1}^{j} e_{i}, j \in[k-1]$ are feasible. To this end, as $P$ is infeasible, the sequence $(1, \ldots, k)$ is valid for $\mathbf{0}$. Consider the valid pair $\left[\mathbf{0}, e_{n}\right]$ and the valid sequence $(1, \ldots, k)$. Let

$$
\begin{aligned}
\left(t_{1}, \ldots, t_{k}\right) & :=T\left[\mathbf{0}, e_{n}\right](1, \ldots, k) \\
y & :=\operatorname{im}\left[\mathbf{0}, e_{n}\right](1, \ldots, k)=e_{n}+\sum_{i=1}^{k} t_{i} e_{i} .
\end{aligned}
$$

By Remark 4.9, $y$ is a feasible point seen by $\mathbf{0}+\sum_{i=1}^{k} e_{i}=b$.

Claim. $y=e_{n}+\sum_{i=1}^{k} e_{i}$.

Proof of Claim. We know that $b$ sees $y$, and clearly $b$ sees $b \triangle e_{n}=e_{n}+\sum_{i=1}^{k} e_{i}$, too. In particular, $y \triangle b$ and $\left(b \triangle e_{n}\right) \triangle b=e_{n}$ are either equal or incomparable. However, as $(y \triangle b)_{n}=1$, it follows that $y \triangle b \geq e_{n}$, implying in turn that $y \triangle b=e_{n}$, so $y=b \triangle e_{n}$, thereby proving the claim.

As an immediate consequence, $t_{1}=t_{2}=\cdots=t_{k}=1$. Take a coordinate $j \in[k-1]$. Then the image of the valid pair $\left[\mathbf{0}, e_{n}\right]$ along the valid sequence $(1, \ldots, j)$ for $\mathbf{0}$ is

$$
\operatorname{im}\left[\mathbf{0}, e_{n}\right](1, \ldots, j)=e_{n}+\sum_{i=1}^{j} t_{i} e_{i}=e_{n}+\sum_{i=1}^{j} e_{i} .
$$

Thus, by Remark 4.9, $e_{n}+\sum_{i=1}^{j} e_{i}$ is feasible, as required.

\subsection{Global implications for resistant sets}

Applying the Plane Propagation Lemma Let us prove Theorem 1.14, stating that for a set $S \subseteq\{0,1\}^{n}$, (i) $S$ is resistant and strictly polar if, and only if, (ii) in every restriction of $S$, either there are antipodal feasible points or the feasible points form a hypercube.

Proof of Theorem 1.14. (i) $\Rightarrow$ (ii): Assume that $S$ is resistant and strictly polar. By Remark 3.2, every restriction of $S$ is also resistant, and by definition, every restriction of $S$ is also strictly polar. Thus, it suffices to show that either $S$ has antipodal points or $S$ is a hypercube. To this end, assume that $S$ does not have antipodal points. As $S$ is polar, the points in $S$ must all agree on a coordinate. The Plane Propagation Lemma now implies that $S$ is a hypercube, as required. (ii) $\Rightarrow$ (i): Assume that in every restriction of $S$,

$(\star)$ there are either antipodal feasible points or the feasible points form a hypercube. 
Obviously, every restriction of $S$ is polar, so $S$ is strictly polar. It remains to show that $S$ is resistant. By Theorem 1.8 (ii), it suffices to show that $S$ has no fragile or $\left\{\mathbf{0}^{k}, \mathbf{1}^{k}-e_{1}\right\}, k \geq 4$ restriction, and as these restrictions do not satisfy $(\star)$, we are done.

Applying the Sight Propagation Lemma The first application of this lemma is the following theorem that we will use in $\S 7$ :

Theorem 4.11. Take an integer $n \geq 1$ and a nonempty resistant set $S \subseteq\{0,1\}^{n}$. Then the following statements hold:

(1) Let $x, x \triangle e_{i}$ be infeasible points for some coordinate $i \in[n]$, and let $y^{1}, y^{2}$ be distinct feasible points seen by $x$. Then $\operatorname{im}\left[x, y^{1}\right](i)$ and $\operatorname{im}\left[x, y^{2}\right](i)$ are distinct points.

(2) For every infeasible component $K$, there is a constant $m_{K} \in\{1 \ldots, n\}$ such that every infeasible point of $K$ sees exactly $m_{K}$ feasible points.

Proof. (1) By definition, $y^{1} \triangle x, y^{2} \triangle x$ are points of minimal support in $S \triangle x$. As $S$ is resistant, $y^{1} \triangle x, y^{2} \triangle x$ have disjoint supports, implying in turn that $\operatorname{dist}\left(y^{1}, y^{2}\right)=\operatorname{dist}\left(y^{1} \triangle x, y^{2} \triangle x\right) \geq 2$. Since $\operatorname{im}\left[x, y^{1}\right](i) \in$ $\left\{y^{1}, y^{1} \triangle e_{i}\right\}$ and $\operatorname{im}\left[x, y^{2}\right](i) \in\left\{y^{2}, y^{2} \triangle e_{i}\right\}$, it follows that $\operatorname{im}\left[x, y^{1}\right](i), \operatorname{im}\left[x, y^{2}\right](i)$ are distinct points. (2) Take neighboring infeasible points $x, x \triangle e_{i}$ and let $m(x), m\left(x \triangle e_{i}\right) \geq 1$ be the number of feasible points $x, x \triangle e_{i}$ see, respectively. It suffices to show that $m(x)=m\left(x \triangle e_{i}\right)$. By symmetry, it actually suffices to show that $m\left(x \triangle e_{i}\right) \geq m(x)$. Let $y^{1}, \ldots, y^{m(x)}$ be the feasible points $x$ sees. By Remark 4.9 and (1), $\operatorname{im}\left[x, y^{1}\right](i), \ldots, \operatorname{im}\left[x, y^{m(x)}\right](i)$ are distinct feasible points seen by $x \triangle e_{i}$. Thus, $m\left(x \triangle e_{i}\right) \geq m(x)$, as required.

For the next application, Theorem 1.15, take an integer $n \geq 3$ and a set $S \subseteq\{0,1\}^{n}$. We say that $S$ is critically non-polar if it is strictly non-polar and, for each $i \in[n]$, both the 0 - and 1-restrictions of $S$ at coordinate $i$ have antipodal points. The Plane and Sight Propagation Lemmas have the following implication:

Proposition 4.12. A resistant strictly non-polar set is critically non-polar.

Proof. Take an integer $n \geq 3$ and a resistant strictly non-polar set $S \subseteq\{0,1\}^{n}$. Suppose for a contradiction that $S$ is not critically non-polar. After twisting and relabeling, if necessary, we may assume that $S_{1}$, the 1restriction of $S$ at coordinate 1 , does not have antipodal points. As $S$ is strictly non-polar, $S_{1}$ is polar so its points must agree on a coordinate. Since it is resistant, the Plane Propagation Lemma implies that $S_{1}$ is a hypercube. After twisting and relabeling, if necessary, we may assume that

$$
(\diamond) \quad S \cap\left\{x: x_{1}=1\right\}=\left\{x \in\{0,1\}^{n}: x_{1}=x_{2}=\cdots=x_{k}=1\right\}
$$

for some $k \in\{2,3, \ldots, n\}$.

Claim. $S \cap\left\{x_{1}=0\right\} \subseteq\left\{x \in\{0,1\}^{n}: x_{2}=\cdots=x_{k}=1\right\}$. 
Proof of Claim. Take a point $b \in\{0,1\}^{n}$ such that $b_{1}=0$ and $b \notin\left\{x: x_{2}=\cdots=x_{k}=1\right\}$. We need to show that $b$ is infeasible. To this end, let $a, c$ be the points in $\left\{x: x_{1}=0, x_{2}=\cdots=x_{k}=0\right\},\left\{x: x_{1}=0, x_{2}=\right.$ $\left.\cdots=x_{k}=1\right\}$ that otherwise agree with $b$, respectively. So $a_{i}=c_{i}=b_{i}$ for all $i \in[n]-[k]$. By assumption, $b \neq c$. Since $(\diamond)$ holds and $S$ does not have antipodal points, $a$ is infeasible. Thus, as $\left[a+e_{1}, c+e_{1}\right]$ is a valid pair, and $a$ is infeasible, the Sight Propagation Lemma implies that $a$ sees one of $c, c+e_{1}$. As a result, all the points in $\{x: a \leq x \leq c, x \neq c\}$, including $b$, are infeasible, as required.

In particular, the points in $S$ agree on coordinate 2, a contradiction as $S$ is non-polar. Thus, $S$ is critically non-polar.

We will need the following result:

Theorem 4.13 ([3], Theorem 3.6). Take an integer $n \geq 3$ and a strictly non-polar set $S \subseteq\{0,1\}^{n}$. Then the following statements are equivalent:

(i) $\operatorname{cuboid}(S)$ is minimally non-packing,

(ii) $S$ is critically non-polar, and every induced clutter of $S$ has the packing property.

We are now ready to prove Theorem 1.15, stating that a resistant set is strictly non-polar if and only if its cuboid is an ideal minimally non-packing clutter.

Proof of Theorem 1.15. Take an integer $n \geq 3$ and let $S \subseteq\{0,1\}^{n}$ be a resistant set. Then by Corollary 1.5, cuboid $(S)$ is ideal. $(\Rightarrow)$ Assume that $S$ is strictly non-polar. By Proposition 4.12, $S$ is critically non-polar, and by Remark 1.11, every induced clutter of $S$ has the packing property. Thus, Theorem 4.13 implies that $\operatorname{cuboid}(S)$ is minimally non-packing, as required. $(\Leftarrow)$ Assume that cuboid $(S)$ is minimally non-packing. Then by Theorem 4.13, $S$ is critically non-polar, so $S$ is strictly non-polar also, thereby finishing the proof.

Applying the Path Propagation Lemma Finally, let us see the following application of this lemma, which will be useful later:

Theorem 4.14. Take an integer $n \geq 3$ and a resistant strictly non-polar set $S \subseteq\{0,1\}^{n}$. Then $\bar{S}$ and $S$ are not strictly connected.

Proof. By Proposition 4.12, $S$ is critically non-polar. Thus, after a possible twisting, we may assume that $\mathbf{0}, \mathbf{1}-e_{n} \in S$. As $S$ does not contain antipodal points, we have that $e_{n}, \mathbf{1} \in \bar{S}$.

Claim 1. $\bar{S}$ is not strictly connected.

Proof of Claim. Suppose for a contradiction that $\bar{S}$ is strictly connected. We will show that $(\star)$ for every infeasible point $a$ in $\left\{x \in\{0,1\}^{n}: x_{n}=1\right\}$, the point $a \triangle e_{n}$ is feasible. 
To this end, as $\bar{S}$ is strictly connected, there exist a straight infeasible $\left(e_{n}, a\right)$-path $P$ and a straight infeasible $(a, \mathbf{1})$-path $Q$, by Proposition 4.3. Since $\operatorname{dist}\left(e_{n}, a\right)+\operatorname{dist}(a, \mathbf{1})=\operatorname{dist}\left(e_{n}, \mathbf{1}\right)$, it follows from Remark 4.2 that $P \cup Q$ is a straight infeasible $\left(e_{n}, \mathbf{1}\right)$-path, which contains $a$. Thus, as $\mathbf{0 , 1}-e_{n}$ are feasible, the Path Propagation Lemma implies that $a \triangle e_{n}$ is feasible, thereby proving $(\star) .(\star)$ implies in particular that every infeasible path is contained in either $\left\{x: x_{n}=1\right\}$ or $\left\{x: x_{n}=0\right\}$. Applying Proposition 4.3, we see that in fact, $\bar{S} \subseteq\left\{x: x_{n}=1\right\}$. Since $S$ does not contain antipodal points, we must have that $\bar{S}=\left\{x: x_{n}=1\right\}$ and $S=\left\{x: x_{n}=0\right\}$, implying in turn that $S$ is polar, a contradiction.

Claim 2. $S$ is not strictly connected.

Proof of Claim. Suppose for a contradiction that $S$ is strictly connected. We will show that

$(\diamond)$ for every feasible point $b$ in $\left\{x \in\{0,1\}^{n}: x_{n}=0\right\}$, the point $b \triangle e_{n}$ is infeasible.

To this end, as $S$ is strictly connected, there exist a straight feasible $(\mathbf{0}, b)$-path $P$ as well as a straight feasible $\left(b, \mathbf{1}-e_{n}\right)$-path $Q$, by Proposition 4.3. Since $\operatorname{dist}(\mathbf{0}, b)+\operatorname{dist}\left(b, \mathbf{1}-e_{n}\right)=\operatorname{dist}\left(\mathbf{0}, \mathbf{1}-e_{n}\right)$, it follows from Remark 4.2 that $P \cup Q$ is a straight feasible $\left(\mathbf{0}, \mathbf{1}-e_{n}\right)$-path, which contains $b$. Since $S$ does not have antipodal points, it follows that $(P \cup Q) \triangle \mathbf{1}$ is a straight infeasible $\left(e_{n}, \mathbf{1}\right)$-path. ${ }^{6}$ The Path Propagation Lemma now implies that $(P \cup Q) \triangle \mathbf{1} \triangle e_{n}$ is a straight feasible $\left(\mathbf{0}, \mathbf{1}-e_{n}\right)$-path. Once again, as $S$ does not have antipodal points, we get that $(P \cup Q) \triangle e_{n}$ is a straight infeasible $\left(e_{n}, \mathbf{1}\right)$-path, implying in particular that $b \triangle e_{n}$ is infeasible, thereby proving $(\diamond) .(\diamond)$ implies in particular that every feasible path is contained in either $\left\{x: x_{n}=0\right\}$ or $\left\{x: x_{n}=1\right\}$. Applying Proposition 4.3, we see that in fact, $S \subseteq\left\{x: x_{n}=0\right\}$, implying in turn that $S$ is polar, a contradiction.

Claims 1 and 2 finish the proof of Theorem 4.14.

As a consequence,

Corollary 4.15. Take an integer $n \geq 1$ and a resistant set $S \subseteq\{0,1\}^{n}$. If $\bar{S}$ or $S$ is strictly connected, then $S$ is strictly polar.

Proof. Let us prove the contrapositive statement. Assume that $S$ is not strictly polar. Then it has a restriction $S^{\prime}$ that is strictly non-polar. We know that $S^{\prime}$ is resistant. It therefore follows from Theorem 4.14 that neither $S^{\prime}$ nor $\overline{S^{\prime}}$ is strictly connected, implying in turn that neither $S$ nor $\bar{S}$ is strictly connected, as required.

\section{Straight circuits}

Take an integer $n \geq 2$. Let $C$ be a circuit of $G_{n}$ whose vertices, denoted $V(C)$, are $v_{0}, v_{1}, \ldots, v_{k}$ in clockwise order. We will represent $C$ as the sequence $\left(v_{0}, v_{1}, \ldots, v_{k}, v_{0}\right)$. Take a point $v \in\{0,1\}^{n}$, an integer $\ell \in$

\footnotetext{
${ }^{6}$ If $P$ is an $(a, b)$-path, then $P \triangle x$ denotes the $(a \triangle x, b \triangle x)$-path whose vertices are the vertices of $P$ twisted by $x$.
} 
$\{2, \ldots, n\}$, and distinct coordinates $i_{1}, \ldots, i_{\ell} \in[n]$. Denote by $\left(v: i_{1}, i_{2}, \ldots, i_{\ell}\right)$ the circuit

$$
\left(v_{0}, v_{1}, \ldots, v_{\ell}, \ldots, v_{2 \ell-1}, v_{2 \ell}=v_{0}\right)
$$

where $v_{0}=v$ and $v_{j}=v_{j-1} \triangle e_{i_{j}}$ and $v_{\ell+j}=v_{\ell+j-1} \triangle e_{i_{j}}$ for each $j \in[\ell]$. We will refer to $\left(v: i_{1}, i_{2}, \ldots, i_{\ell}\right)$ as a straight circuit. (Notice that any point of the straight circuit can be a starting point.)

The length of a circuit is the number of edges it has. Take a set $S \subseteq\{0,1\}^{n}$. We refer to every circuit of $G_{n}[S]$ as a feasible circuit and to every circuit of $G_{n}[\bar{S}]$ as an infeasible circuit. The purpose of this section is to prove the following statement:

Take an integer $n \geq 4$ and a resistant set $S \subseteq\{0,1\}^{n}$ that is non-polar. Assume that there is a straight infeasible circuit $K$ of length $2(n-1)$ contained in $\left\{x: x_{n}=0\right\}$ such that $V\left(K \triangle e_{n}\right) \subseteq S$.

Then $S$ has one of $\left\{R_{k, 1}: k \geq 1\right\} \cup\left\{R_{5}\right\}$ as a restriction. ${ }^{7}$

This tool is crucial for proving the main result of the paper, Theorem 1.17. To prove this statement, let us start with the following lemma that is widely referenced throughout this section:

Lemma 5.1 (Straight Circuit). Take an integer $n \geq 4$ and a resistant set $S \subseteq\{0,1\}^{n}$ without antipodal points. Let $K$ be a straight infeasible circuit of length $2(n-1)$ contained in $\left\{x: x_{n}=0\right\}$ such that $V\left(K \triangle e_{n}\right) \subseteq S$. Then for a vertex $v \in\left\{x: x_{n}=0\right\}-V(K)$ that is adjacent to a vertex of $K$, either

$$
\left\{v, \mathbf{1}-v \triangle e_{n}\right\} \subseteq \bar{S} \text { and }\left\{v \triangle e_{n}, \mathbf{1}-v\right\} \subseteq S
$$

or

$$
\left\{v, \mathbf{1}-v \triangle e_{n}\right\} \subseteq S \quad \text { and } \quad\left\{v \triangle e_{n}, \mathbf{1}-v\right\} \subseteq \bar{S}
$$

Proof. After a possible relabeling and twisting, we may assume that

$$
K=(\mathbf{0}: 1,2, \ldots, n-1)=\left(v_{0}, v_{1}, \ldots, v_{n-1}, \ldots, v_{2 n-3}, v_{2 n-2}=v_{0}\right),
$$

where $v_{0}=\mathbf{0}$ and $v_{j}=v_{j-1} \triangle e_{j}$ and $v_{n-1+j}=v_{n-1+j-1} \triangle e_{j}$ for each $j \in[n-1]$.

Claim. Take a vertex $w \in\left\{x: x_{n}=0\right\}-V(K)$ that is adjacent to a vertex of $K$. If $w \in \bar{S}$ then $w \triangle e_{n} \in S$.

Proof of Claim. By the symmetry between the vertices of $K$, we may assume that $w$ is adjacent to $v_{0}$, that is, $w=v_{0} \triangle e_{i}$ for some $i \in[n-1]-\{1, n-1\}$. Let

$$
P:=\left(v_{n-1+i}, v_{n-1+i+1}, \ldots, v_{2 n-2}=v_{0}\right) \quad \text { and } \quad Q:=\left(v_{0}, v_{1}, \ldots, v_{i-1}\right) \quad \text { and } \quad R:=P \cup Q .
$$

Notice that $R$ is a straight subpath of the infeasible circuit $K$. The Path Propagation Lemma implies that the feasible points in $R \triangle e_{i}$ should form a path. Thus, since $v_{0} \triangle e_{i}=w \in \bar{S}$, it follows that either $V\left(P \triangle e_{i}\right) \subseteq \bar{S}$ or $V\left(Q \triangle e_{i}\right) \subseteq \bar{S}$. By symmetry, we may assume that $V\left(P \triangle e_{i}\right) \subseteq \bar{S}$. Consider now the straight infeasible path $P^{\prime}:=\left(v_{0}\right) \cup\left[P \triangle e_{i}\right]$ whose ends are $v_{0}$ and $v_{n-1+i} \triangle e_{i}=v_{n-1+i-1}$. Since $\left\{v_{0} \triangle e_{n}, v_{n-1+i-1} \triangle e_{n}\right\} \subseteq$ $V\left(K \triangle e_{n}\right) \subseteq S$, it follows from the Path Propagation Lemma that $P^{\prime} \triangle e_{n}$ is a feasible path. In particular, we have that $v_{0} \triangle e_{i} \triangle e_{n}=w \triangle e_{n} \in S$.

\footnotetext{
${ }^{7}$ If $C$ is a circuit, then $C \triangle x$ denotes the circuit whose vertices are the vertices of $C$ twisted by $x$.
} 
Now take a vertex $v \in\left\{x: x_{n}=0\right\}-V(K)$ that is adjacent to a vertex of $K$. Assume in the first case that $v \in \bar{S}$. By the claim, $v \triangle e_{n} \in S$. Since $S$ does not contain antipodal points, we get that $1-v \triangle e_{n} \in \bar{S}$. Since $\mathbf{1}-v \triangle e_{n}$ is also adjacent to a vertex of $K$, and is not a vertex of $K$, it follows from the claim that $\mathbf{1}-v \in S$. Assume in the remaining case that $v \in S$. As $S$ does not contain antipodal points, $1-v \in \bar{S}$, so by the claim, we have $1-v \triangle e_{n} \in S$, so its antipodal point $v \triangle e_{n}$ belongs to $\bar{S}$. This finishes the proof of the lemma.

Before moving on, we should point out that the results in this section will make heavy use of the Sight Propagation Lemma, most often applied as illustrated in the following figure,

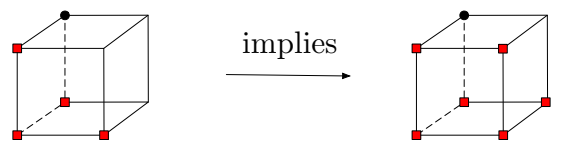

and in most cases, we will leave it to the reader to identify the 3-dimensional cube where the Sight Propagation Lemma is being applied.

\subsection{When there are no $R_{1,1}, R_{5}$ restrictions}

We will need the following technical lemma:

Lemma 5.2. Take an integer $n \geq 6$ and a resistant set $S \subseteq\{0,1\}^{n}$ without antipodal points and without an $R_{1,1}, R_{5}$ restriction. Suppose $K:=(\mathbf{0}: 1,2,3,4,5, \ldots, n-1)$ is a straight infeasible circuit, $V\left(K \triangle e_{n}\right) \subseteq S$, and $\left\{e_{2}, e_{2}+e_{3}, e_{3}, e_{1}+e_{3}\right\} \subseteq S$. Then, for each $i \in[n-4]$,

$$
K_{i}:=(\mathbf{0}: 4, \ldots, 3+i, 1,2,3,3+i+1, \ldots, n-1)
$$

is a straight infeasible circuit, $V\left(K_{i} \triangle e_{n}\right) \subseteq S$ and $\left\{e_{2}, e_{2}+e_{3}, e_{3}, e_{1}+e_{3}\right\} \triangle e_{4} \triangle \cdots \triangle e_{3+i} \subseteq S$.

Proof. We proceed by induction on $i \geq 1$. Let us first prove the base case $i=1$, which we restate as follows:

$(\star)$ Take an integer $n \geq 6$ and a resistant set $S \subseteq\{0,1\}^{n}$ without antipodal points and without an $R_{1,1}, R_{5}$ restriction. Suppose $K:=(0: 1,2,3,4,5, \ldots, n-1)$ is a straight infeasible circuit, $V\left(K \triangle e_{n}\right) \subseteq S$, and $\left\{e_{2}, e_{2}+e_{3}, e_{3}, e_{1}+e_{3}\right\} \subseteq S$. Then, $K^{\prime}:=(\mathbf{0}: 4,1,2,3,5, \ldots, n-1)$ is a straight infeasible circuit, $V\left(K^{\prime} \triangle e_{n}\right) \subseteq S$ and $\left\{e_{2}, e_{2}+e_{3}, e_{3}, e_{1}+e_{3}\right\} \triangle e_{4} \subseteq S$.

Define $P_{0}, P_{1}, Q_{0}, Q_{1} \subseteq\{0,1\}^{4}$ as follows: $P_{0}$ (resp. $P_{1}$ ) is obtained after 0 -restricting coordinates $5, \ldots, n-1$ and 0-restricting coordinate $n$ (resp. 1-restricting coordinate $n$ ), and $Q_{0}$ (resp. $Q_{1}$ ) is obtained after 1-restricting coordinates $5, \ldots, n-1$ and 0 -restricting coordinate $n$ (resp. 1-restricting coordinate $n$ ). Since $V(K) \subseteq \bar{S}$ and $V\left(K \triangle e_{n}\right) \subseteq S$, it follows that

$$
\begin{aligned}
\{0000,1000,1100,1110,1111\} \subseteq \overline{P_{0}} & \{0000,1000,1100,1110,1111\} \subseteq P_{1} \\
\{1111,0111,0011,0001,0000\} \subseteq \overline{Q_{0}} & \{1111,0111,0011,0001,0000\} \subseteq Q_{1} .
\end{aligned}
$$


By assumption, we also know that

$$
\{0100,0110,0010,1010\} \subseteq P_{0} .
$$

In $\{0,1\}^{n}$, each one of these points belongs to $\bar{K}$ and is adjacent to a vertex of $K$, so by the Straight Circuit Lemma,

$$
\begin{aligned}
& \{0100,0110,0010,1010\} \subseteq \overline{P_{1}} \\
& \{1011,1001,1101,0101\} \subseteq Q_{0} \\
& \{1011,1001,1101,0101\} \subseteq \overline{Q_{1}} .
\end{aligned}
$$

See the following figure illustrating the inclusions listed so far:
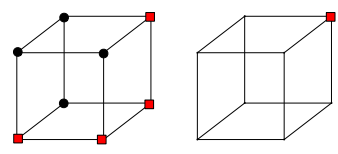

$P_{0}$
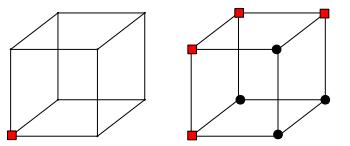

$Q_{0}$
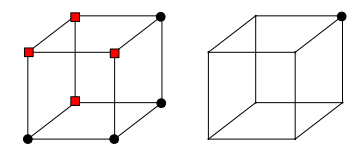

$P_{1}$
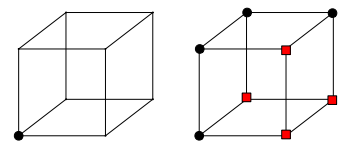

$Q_{1}$

In the following claim, we will take advantage of the assumption that $S$ has no $R_{1,1}, R_{5}$ restriction.

Claim 1. $\{0001,1001,1101\} \subseteq \overline{P_{0}} \cap P_{1}$ and $\{1110,0110,0010\} \subseteq \overline{Q_{0}} \cap Q_{1}$.

Proof of Claim. We will first show that $1101 \in \overline{P_{0}}$. Suppose for a contradiction that $1101 \in P_{0}$. Since $S$ is resistant, it follows from the Sight Propagation Lemma that $1001 \in P_{0}$. As the vertices in $\{0,1\}^{n}$ corresponding to 1101,1001 are in $\bar{K}$ and adjacent to vertices of $K$, the Straight Circuit Lemma implies that $\{1101,1001\} \subseteq \overline{P_{1}}$ and $\{0010,0110\} \subseteq Q_{0} \cap \overline{Q_{1}}$. Since the restriction of $\left(P_{0} \times\{0\}\right) \cup\left(P_{1} \times\{1\}\right)$ obtained after 1-restricting coordinate 2 and 0-restricting coordinate 3 is neither $P_{3}$ nor $R_{1,1}$, it follows that $0101 \in P_{0}$. As $S$ does not contain antipodal points, we get that $1010 \in \overline{Q_{1}}$ :
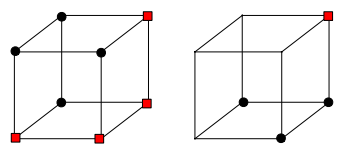

$P_{0}$
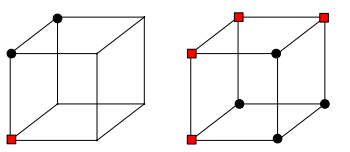

$Q_{0}$
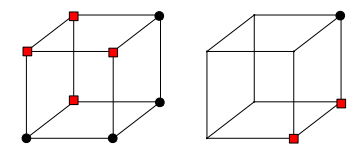

$P_{1}$
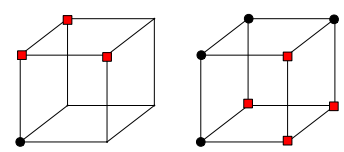

$Q_{1}$ 
Since the 0-restriction of $Q_{1}$ at coordinate 2 is resistant, it follows that $1000 \in Q_{1}$, so by the Straight Circuit Lemma, $1000 \in \overline{Q_{0}}$ and $0111 \in \overline{P_{0}} \cap P_{1}$. As the 1-restriction of $Q_{1}$ at coordinate 3 is resistant, we have $1110 \in Q_{1}$, and so by the Straight Circuit Lemma, $1110 \in \overline{Q_{0}}$ and $0001 \in \overline{P_{0}} \cap P_{1}$ :

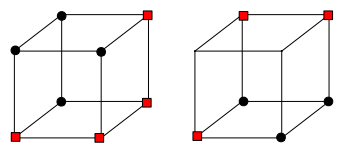

$P_{0}$

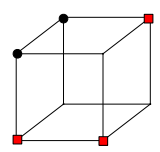

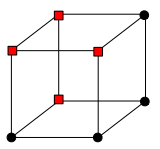

$P_{1}$
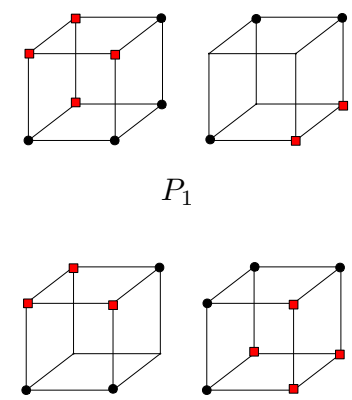

$Q_{1}$

Since the 1-restriction of $P_{0}$ at coordinate 3 is resistant, we get that $1011 \in P_{0}$, and by the Straight Circuit Lemma, $1011 \in \overline{P_{1}}$ and $0100 \in Q_{0} \cap \overline{Q_{1}}$. As the restriction of $\left(Q_{0} \times\{0\}\right) \cup\left(Q_{1} \times\{1\}\right)$ obtained after 1-restricting coordinate 1 and 0-restricting coordinate 4 is resistant and has no $R_{1,1}$ restriction, it follows that $1100 \in Q_{1}$ and $1010 \in Q_{0}$. Since $S$ does not have antipodal points, we get that $0011 \in \overline{P_{0}}$ and $0101 \in \overline{P_{1}}$ :
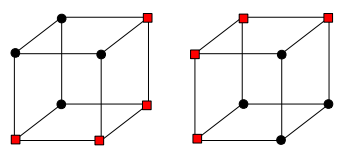

$P_{0}$
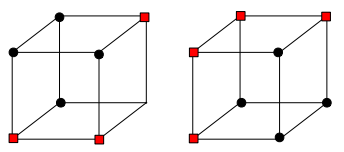

$Q_{0}$
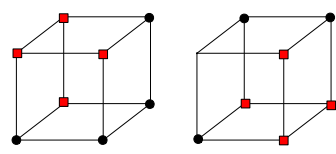

$P_{1}$
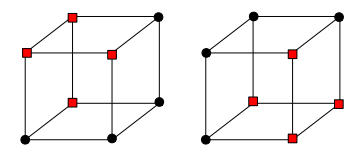

$Q_{1}$

Since the 0-restriction of $P_{1}$ at coordinate 2 is resistant, it follows that $0011 \in P_{1}$, implying in turn that $\left(P_{0} \times\right.$ $\{0\}) \cup\left(P_{1} \times\{1\}\right) \cong R_{5}$, so $S$ has an $R_{5}$ restriction, a contradiction. Thus, $1101 \in \overline{P_{0}}$.

It follows from the Sight Propagation Lemma that $\{1001,0001\} \subseteq \overline{P_{0}}$. By the Straight Circuit Lemma, $\{0001,1001,1101\} \subseteq P_{1}$ and $\{1110,0110,0010\} \subseteq \overline{Q_{0}} \cap Q_{1}$, as claimed.

Recall that $K^{\prime}=(\mathbf{0}: 4,1,2,3,5, \ldots, n-1)$. Notice that by Claim $1, K^{\prime}$ is a straight infeasible circuit such that $V\left(K^{\prime} \triangle e_{n}\right) \subseteq S$. In the following claim, we will apply the Straight Circuit Lemma to the straight infeasible circuit $K^{\prime}$.

Claim 2. $\{0101,0111,0011,1011\} \subseteq P_{0}$.

Proof of Claim. Since $P_{1}$ is resistant, it follows from the Sight Propagation Lemma that either

$$
\{0011,0111\} \subseteq P_{1} \quad \text { or } \quad\{0011,0111\} \subseteq \overline{P_{1}} .
$$


We claim that $\{0011,0111\} \subseteq \overline{P_{1}}$. Suppose for a contradiction that $\{0011,0111\} \subseteq P_{1}$. After applying the Straight Circuit Lemma to $K^{\prime}$, we get that $\{0011,0111\} \subseteq \overline{P_{0}}$ :

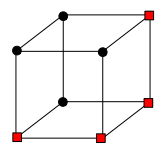

$P_{0}$

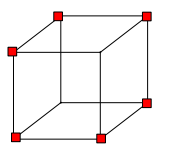

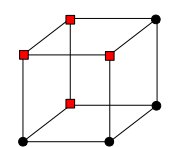

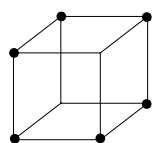

$P_{1}$

Since $P_{0}$ is resistant, it follows from Theorem 1.8 (ii) that $P_{0}$ has no fragile restriction. However, either its 0restriction at coordinate 2 or its 1 -restriction at coordinate 3 is fragile, a contradiction. Thus, $\{0011,0111\} \subseteq \overline{P_{1}}$. After applying the Straight Circuit Lemma to $K^{\prime}$, we get that $\{0011,0111\} \subseteq P_{0}$. As $P_{0}$ is resistant, it follows that $\{0101,1011\} \subseteq P_{0}$, as required.

By Claim 2, $\left\{e_{2}, e_{2}+e_{3}, e_{3}, e_{1}+e_{3}\right\} \triangle e_{4} \subseteq S$. This proves $(\star)$ and in turn the base case $i=1$. For the induction step, assume that $i \geq 2$. Then an application of $(\star)$ to $K_{i-1}$, instead of $K$, implies that $K_{i}:=$ $(\mathbf{0}: 4, \ldots, 3+i, 1,2,3,3+i+1, \ldots, n-1)$ is a straight infeasible circuit, $V\left(K_{i} \triangle e_{n}\right) \subseteq S$, and $\left\{e_{2}, e_{2}+\right.$ $\left.e_{3}, e_{3}, e_{1}+e_{3}\right\} \triangle e_{4} \triangle \cdots \triangle e_{3+i} \subseteq S$, thereby completing the induction step.

Let $D_{3}:=\{000,100,110,111\} \subseteq\{0,1\}^{3}$. Using the preceding lemma, we prove the following:

Proposition 5.3. Take an integer $n \geq 5$ and a resistant set $S \subseteq\{0,1\}^{n}$ without antipodal points and without an $R_{1,1}, R_{5}$ restriction. Let $K$ be a straight infeasible circuit of length $2(n-1)$ contained in $\left\{x: x_{n}=0\right\}$ such that $V\left(K \triangle e_{n}\right) \subseteq S$. Then $S$ does not have a $D_{3}$ restriction whose infeasible points all belong to $K$.

Proof. After a possible relabeling and twisting, we may assume that $K=(\mathbf{0}: 1,2, \ldots, n-1)$. Suppose for a contradiction that $S$ has a $D_{3}$ restriction whose infeasible points all belong to $K$. By symmetry, we may assume that the $D_{3}$ restriction is obtained after 0-restricting coordinates $4, \ldots, n$, that is, $\left\{e_{2}, e_{2}+e_{3}, e_{3}, e_{1}+e_{3}\right\} \subseteq S$. Assume in the first case that $n \geq 6$. It then follows from Lemma 5.2 that for each $i \in[n-4], K_{i}:=$ $(\mathbf{0}: 4, \ldots, 3+i, 1,2,3,3+i+1, \ldots, n-1)$ is a straight infeasible circuit, $V\left(K_{i} \triangle e_{n}\right) \subseteq S$, and $\left\{e_{2}, e_{2}+\right.$ $\left.e_{3}, e_{3}, e_{1}+e_{3}\right\} \triangle e_{4} \triangle \cdots \triangle e_{3+i} \subseteq S$. In particular, setting $i=n-4$, we get that

$$
e_{3} \triangle e_{4} \triangle e_{5} \triangle \cdots \triangle e_{n-1} \in S
$$

However, $e_{3} \triangle e_{4} \triangle e_{5} \Delta \cdots \Delta e_{n-1} \in K \subseteq \bar{S}$, a contradiction. Assume in the remaining case that $n=5$. Let $P_{0} \subseteq\{0,1\}^{4}$ (resp. $P_{1} \subseteq\{0,1\}^{4}$ ) be the 0-restriction (resp. 1-restriction) of $S$ at coordinate 5. Since $V(K) \subseteq \bar{S}$ and $V\left(K \triangle e_{n}\right) \subseteq S$, it follows that

$$
\begin{aligned}
& \{0000,1000,1100,1110,1111,0111,0011,0001\} \subseteq \overline{P_{0}} \\
& \{0000,1000,1100,1110,1111,0111,0011,0001\} \subseteq P_{1}
\end{aligned}
$$

As the 0 -restriction of $P_{0}$ at coordinate 4 yields $D_{3}$, we also know that $\{0100,0110,0010,1010\} \subseteq P_{0}$. In $\{0,1\}^{5}$, each one of these points belongs to $\bar{K}$ and is adjacent to a vertex of $K$, so by the Straight Circuit 
Lemma,

$$
\begin{aligned}
& \{0100,0110,0010,1010\} \subseteq \overline{P_{1}} \\
& \{1011,1001,1101,0101\} \subseteq P_{0} \\
& \{1011,1001,1101,0101\} \subseteq \overline{P_{1}} .
\end{aligned}
$$

The points given above determine that $S \cong R_{5}$,
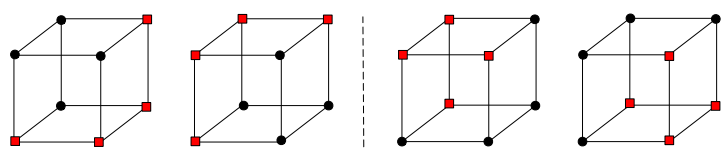

$P_{0}$

$P_{1}$

$S$

a contradiction.

\subsection{Finding $\left\{R_{k, 1}: k \geq 1\right\} \cup\left\{R_{5}\right\}$ restrictions}

For an integer $n \geq 3$, we will need the following property defined on the points $x$ in $\{0,1\}^{n}$ :

$(\diamond) x$ is feasible $\Leftrightarrow \mathbf{1}-x \triangle e_{n}$ is feasible $\Leftrightarrow x \triangle e_{n}$ is infeasible $\Leftrightarrow \mathbf{1}-x$ is infeasible.

Lemma 5.4. Take an integer $n \geq 5$ and a resistant set $S \subseteq\{0,1\}^{n}$ that does not have antipodal points, and let $K:=(\mathbf{0}: 1,2, \ldots, n-1)$ be a straight infeasible circuit such that $V\left(K \triangle e_{n}\right) \subseteq S$. Suppose that $\ell \in\{2, \ldots, n-3\}$ is an integer such that the points in $\left\{x: x_{\ell+1}=\cdots=x_{n}=0\right\}$ satisfy $(\diamond)$ and the feasible points in there form a hypercube. Then one of the following statements hold:

- $S$ has one of $\left\{R_{k, 1}: 1 \leq k \leq \ell\right\} \cup\left\{R_{5}\right\}$ as a restriction, or

- the points in $\left\{x: x_{\ell+2}=\cdots=x_{n}=0\right\}$ satisfy $(\diamond)$ and the feasible points in there form a hypercube.

Proof. Let us proceed by induction on $\ell \geq 2$.

For the base case, assume that $\ell=2$. Notice that every point in $\left\{x: x_{4}=\cdots=x_{n}=0\right\}$ either belongs to $K$ or is adjacent to a vertex of $K$. It therefore follows from the Straight Circuit Lemma that every point in $\left\{x: x_{4}=\cdots=x_{n}=0\right\}$ satisfies $(\diamond)$. Suppose that the feasible points in $\left\{x: x_{4}=\cdots=x_{n}=0\right\}$ do not form a hypercube. Let $H \subseteq\{0,1\}^{4}$ be the 0 -restriction of $S$ at coordinates $4, \ldots, n-1$. Since $V(K) \subseteq \bar{S}$ and $V\left(K \triangle e_{n}\right) \subseteq S$, we see that $\{0000,1000,1100,1110\} \subseteq \bar{H}$ and $\{0001,1001,1101,1111\} \subseteq H$. As the 0-restriction of $H$ at the last coordinate is not a hypercube, one of the following inclusions must hold:

- $\{0100,1010\} \subseteq H$ : By $(\diamond),\{0101,1011\} \subseteq \bar{H}$. If $|\{0010,0110\} \cap H|=0$, then by $(\diamond), H \cong R_{2,1}$, so $S$ has an $R_{2,1}$ restriction. If $|\{0010,0110\} \cap H|=1$, then by $(\diamond), H$, and therefore $S$, has an $R_{1,1}$ restriction. Otherwise, when $|\{0010,0110\} \cap H|=2$, then $H \cong D_{3}$ and so $S$ has a $D_{3}$ restriction whose infeasible points all belong to $K$, so by Proposition 5.3, $S$ has one of $R_{1,1}, R_{5}$ as a restriction. 
- $\{0110,1010\} \subseteq H$ : Since $H$ is resistant, it follows that $0100 \in H$, so by the preceding case, $S$ has one of $R_{1,1}, R_{2,1}, R_{5}$ as a restriction.

- $\{0100,0010\} \subseteq H$ : Since $H$ is resistant, it follows that $1010 \in H$, so by the first case, $S$ has one of $R_{1,1}, R_{2,1}, R_{5}$ as a restriction.

In each case, we see that $S$ has one of $R_{1,1}, R_{2,1}, R_{5}$ as a restriction, thereby proving the base case $\ell=2$.

For the induction step, assume that $\ell \geq 3$. Then $n \geq 6$. Let $S^{\prime}:=S \cap\left\{x: x_{\ell+1}=\cdots=x_{n}=0\right\}$. By assumption, $S^{\prime}$ is a (possibly empty) hypercube, which excludes the points $0, \sum_{i=1}^{\ell} e_{i}$ as these two points belong to the infeasible circuit $K$. Since $S^{\prime}$ is a hypercube and the points in $\left\{x: x_{\ell+1}=\cdots=x_{n}=0\right\}$ satisfy $(\diamond)$,

(1) every infeasible point of $\left\{x: x_{\ell+1}=\cdots=x_{n}=0\right\}$ appears on a straight infeasible circuit $K^{\prime}:=\left(\mathbf{0}: i_{1}, \ldots, i_{\ell}, \ell+1, \ldots, n-1\right)$ such that $V\left(K^{\prime} \triangle e_{n}\right) \subseteq S$, where $i_{1}, \ldots, i_{\ell}$ is some permutation of $1, \ldots, \ell$.

We will use (1) throughout the proof to reroute the circuit $K$. Notice that together with the Straight Circuit Lemma, (1) implies that

(2) every point of $\left\{x: x_{\ell+2}=\cdots=x_{n}=0\right\}$ adjacent to an infeasible point of $\left\{x: x_{\ell+1}=\cdots=\right.$ $\left.x_{n}=0\right\}$ satisfies $(\diamond)$.

As a result, if the feasible points in $\left\{x: x_{\ell+2}=\cdots=x_{n}=0\right\}$ form a hypercube, then every infeasible point in $\left\{x: x_{\ell+2}=\cdots=x_{n}=0\right\}$ satisfies $(\diamond)$, and so every infeasible point of $\left\{x: x_{\ell+2}=\cdots=x_{n}=0\right\}$ appears on a straight infeasible circuit $K^{\prime}:=\left(0: i_{1}, \ldots, i_{\ell+1}, \ell+2, \ldots, n-1\right)$ such that $V\left(K^{\prime} \triangle e_{n}\right) \subseteq S$, where $i_{1}, \ldots, i_{\ell+1}$ is some permutation of $1, \ldots, \ell+1$. Thus, by the Straight Circuit Lemma,

(3) if the feasible points in $\left\{x: x_{\ell+2}=\cdots=x_{n}=0\right\}$ form a hypercube, then every infeasible point in $\left\{x: x_{\ell+2}=\cdots=x_{n}=0\right\}$ satisfies $(\diamond)$.

Suppose that $S$ has none of $\left\{R_{k, 1}: 1 \leq k \leq \ell\right\} \cup\left\{R_{5}\right\}$ as a restriction. By (3), it suffices to show that the feasible points in $\left\{x: x_{\ell+2}=\cdots=x_{n}=0\right\}$ form a hypercube. As $\mathbf{0}, \sum_{i=1}^{\ell} e_{i} \notin S^{\prime}$, it follows that $S^{\prime}$ is a hypercube of dimension at most $\ell-2$. There are five cases:

(i) $S^{\prime}=\emptyset$,

(ii) $S^{\prime}$ is nonempty, of dimension at most $\ell-3$, and has no vertex adjacent to $\sum_{i=1}^{\ell} e_{i}$,

(iii) $S^{\prime}$ is nonempty, of dimension at most $\ell-3$, and has a vertex adjacent to $\sum_{i=1}^{\ell} e_{i}$,

(iv) $S^{\prime}$ is of dimension $\ell-2$ and $\ell=3$,

(v) $S^{\prime}$ is of dimension $\ell-2$ and $\ell \geq 4$. 
(i) In this case, it follows from the Plane Propagation Lemma that the feasible points in $\left\{x: x_{\ell+2}=\cdots=\right.$ $\left.x_{n}=0\right\}$ form a hypercube, thereby completing the induction step.

(ii) In this case, after possibly relabeling coordinates $1, \ldots, \ell$ and rerouting $K$ according to (1), we may assume that $S^{\prime} \subseteq\left\{x \in\{0,1\}^{n}: x_{\ell}=0\right\}$ while $K$ remains as $(\mathbf{0}: 1, \ldots, n-1)$. Consider the following illustration of $\left\{x: x_{\ell+2}=\cdots=x_{n}=0\right\}$ :

$$
x_{\ell}=0 \quad x_{\ell}=1
$$
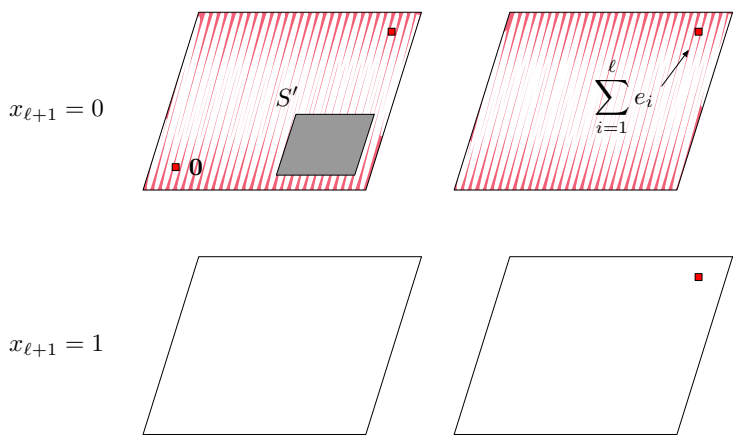

The filled-in parallelogram shows the feasible points of $S^{\prime}$, while the shaded area and the square vertices indicate infeasible points. As $S^{\prime} \neq \emptyset$, the infeasible point $\sum_{i=1}^{\ell} e_{i}$ sees a feasible point in $S^{\prime}$, so by the Sight Propagation Lemma, $\sum_{i=1}^{\ell+1} e_{i}$ sees a feasible point in $S^{\prime} \cup\left(S^{\prime} \triangle e_{\ell+1}\right)$. In particular,

(4) $S^{\prime} \triangle e_{\ell+1} \triangle e_{\ell}$ contains an infeasible point,

and $\sum_{i=1}^{\ell+1} e_{i}-e_{\ell}$ is infeasible, and by the Straight Circuit Lemma, $\sum_{i=1}^{\ell+1} e_{i}-e_{\ell}$ satisfies $(\diamond)$. Consider now the straight infeasible circuit

$$
K^{\prime}:=(\mathbf{0}: 1, \ldots, \ell-1, \ell+1, \ell, \ldots, n-1)
$$

such that $V\left(K^{\prime} \triangle e_{n}\right) \subseteq S$. Let us apply the induction hypothesis to $K^{\prime}$ given that the points in $\left\{x: x_{\ell}=\right.$ $\left.x_{\ell+1}=x_{\ell+2}=\cdots=x_{n}=0\right\}$ satisfy $(\diamond)$ and its feasible points form a hypercube. The induction hypothesis implies that the points in $\left\{x: x_{\ell}=x_{\ell+2}=\cdots=x_{n}=0\right\}$ also satisfy $(\diamond)$ and its feasible points form a hypercube. In particular, by (2), the points in $\left\{x: x_{\ell+2}=\cdots=x_{n}=0\right\}$ all satisfy $(\diamond)$. Moreover, as $S^{\prime} \neq \emptyset$, $S \cap\left\{x: x_{\ell}=x_{\ell+2}=\cdots=x_{n}=0\right\}$ is either $S^{\prime}$ or $S^{\prime} \cup\left(S^{\prime} \triangle e_{\ell+1}\right)$.

Assume in the first case that $S \cap\left\{x: x_{\ell}=x_{\ell+2}=\cdots=x_{n}=0\right\}=S^{\prime}$. We then must have that

$$
S \cap\left\{x: x_{\ell+2}=\cdots=x_{n}=0\right\}=S^{\prime} .
$$

Suppose not. Pick the closest pair of feasible vertices $a, b$ such that $a \in S^{\prime}$ and $b \in\left\{x: x_{\ell+2}=\cdots=x_{n}=\right.$ $0\}-S^{\prime}$. Since the points in $\left\{x: x_{\ell+2}=\cdots=x_{n}=0\right\}$ satisfy $(\diamond)$, it follows that the restriction of $S$ containing $a, b \triangle e_{n}$ as antipodal points is one of $\left\{R_{k, 1}: 1 \leq k \leq \ell\right\}$ as a restriction, a contradiction. Thus, the equation above holds, implying in turn that the feasible points in $\left\{x: x_{\ell+2}=\cdots=x_{n}=0\right\}$ form a hypercube, thereby completing the induction step.

Assume in the remaining case that $S \cap\left\{x: x_{\ell}=x_{\ell+2}=\cdots=x_{n}=0\right\}=S^{\prime} \cup\left(S^{\prime} \triangle e_{\ell+1}\right)$ : 

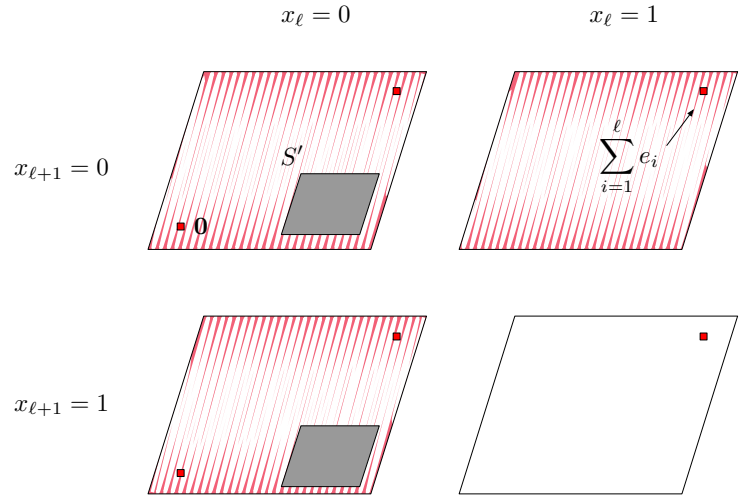

Consider the straight infeasible circuit

$$
K^{\prime \prime}:=\left(e_{\ell+1}: 1, \ldots, \ell, \ell+2, \ldots, n-1, \ell+1\right)
$$

such that $V\left(K^{\prime \prime} \triangle e_{n}\right) \subseteq S$. Let us apply the induction hypothesis to $K^{\prime \prime}$ given that the points in $\left\{x: x_{\ell}=\right.$ $\left.x_{\ell+2}=\cdots=x_{n}=0, x_{\ell+1}=1\right\}$ satisfy $(\diamond)$ and its feasible points form a hypercube. The induction hypothesis implies that the feasible points in $\left\{x: x_{\ell+2}=\cdots=x_{n}=0, x_{\ell+1}=1\right\}$ form a hypercube. That is, $S \cap\{x$ : $\left.x_{\ell+2}=\cdots=x_{n}=0, x_{\ell+1}=1\right\}$ is either $S^{\prime} \triangle e_{\ell+1}$ or $\left(S^{\prime} \triangle e_{\ell+1}\right) \cup\left(S^{\prime} \triangle e_{\ell+1} \triangle e_{\ell}\right)$. However, the latter is not possible by (4), so $S \cap\left\{x: x_{\ell+2}=\cdots=x_{n}=0, x_{\ell+1}=1\right\}=S^{\prime} \triangle e_{\ell+1}$ and

$$
S \cap\left\{x: x_{\ell+2}=\cdots=x_{n}=0\right\}=S^{\prime} \cup\left(S^{\prime} \triangle e_{\ell+1}\right) .
$$

Thus, the feasible points in $\left\{x: x_{\ell+2}=\cdots=x_{n}=0\right\}$ form a hypercube, thereby completing the induction step.

(iii) In this case, as $S^{\prime}$ has dimension at most $\ell-3$, it cannot have a vertex adjacent to $\mathbf{0}$. So, after possibly relabeling coordinates $1, \ldots, \ell$ and rerouting $K$ according to (1), we may assume that $S^{\prime} \subseteq\left\{x: x_{\ell}=1, x_{1}=\right.$ $\left.0, x_{2}=1\right\}$ and $\sum_{i=2}^{\ell} e_{i} \in S^{\prime}$ while $K$ remains as $(0: 1, \ldots, n-1)$ :

$$
x_{\ell}=0
$$

$x_{\ell}=1$
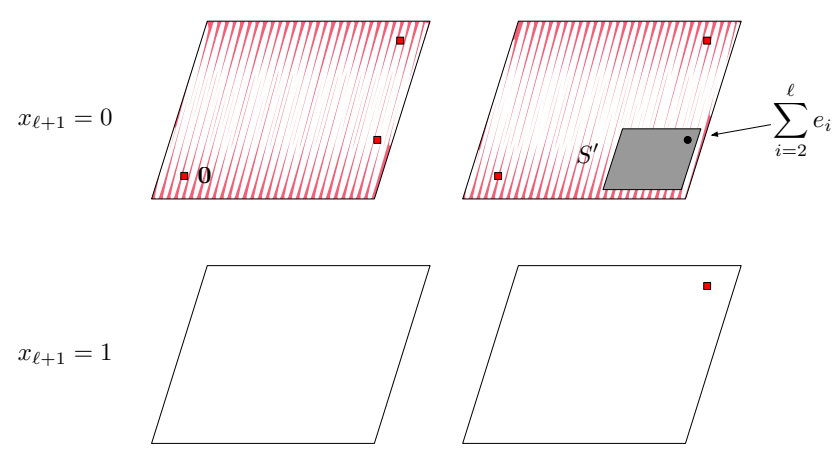

Consider the straight circuit

$$
K^{\prime}:=\left(e_{\ell}: 1, \ldots, \ell-1, \ell+1, \ldots, n-1, \ell\right)
$$


Since $e_{\ell}$ is infeasible and satisfies $(\diamond)$ by (2), it follows that $K^{\prime}$ is infeasible and $K^{\prime} \triangle e_{n}$ is feasible. Let us apply the induction hypothesis to $K^{\prime}$, given that the points in $\left\{x: x_{\ell+1}=x_{\ell+2}=\cdots=x_{n}=0, x_{\ell}=1\right\}$ satisfy ( $\left.\diamond\right)$ and its feasible points form a hypercube. The induction hypothesis implies that the points in $\left\{x: x_{\ell+2}=\cdots=\right.$ $\left.x_{n}=0, x_{\ell}=1\right\}$ satisfy $(\diamond)$ and its feasible points form a hypercube. Together with (2), this implies that all the points in $\left\{x: x_{\ell+2}=\cdots=x_{n}=0\right\}$ satisfy $(\diamond)$.

Assume in the first case that $S \cap\left\{x: x_{\ell+2}=\cdots=x_{n}=0, x_{\ell}=1\right\}=S^{\prime}$. Then we must have that

$$
S \cap\left\{x: x_{\ell+2}=\cdots=x_{n}=0\right\}=S^{\prime} .
$$

Suppose otherwise. Pick a closest pair of feasible points $a, b$ such that $a \in S^{\prime}$ and $b \in\left\{x: x_{\ell+2}=\cdots=x_{n}=\right.$ $0\}-S^{\prime}$. As the points in $\left\{x: x_{\ell+2}=\cdots=x_{n}=0\right\}$ satisfy $(\diamond)$, it follows that the restriction of $S$ containing $a, b \triangle e_{n}$ as antipodal points is one of $\left\{R_{k, 1}: 1 \leq k \leq \ell\right\}$, a contradiction. Thus, $S \cap\left\{x: x_{\ell+2}=\cdots=x_{n}=\right.$ $0\}=S^{\prime}$. So the feasible points in $\left\{x: x_{\ell+2}=\cdots=x_{n}=0\right\}$ form a hypercube, thereby completing the induction step.

Assume in the remaining case that $S \cap\left\{x: x_{\ell+2}=\cdots=x_{n}=0, x_{\ell}=1\right\}=S^{\prime} \cup\left(S^{\prime} \triangle e_{\ell+1}\right)$ :

$$
x_{\ell}=0 \quad x_{\ell}=1
$$
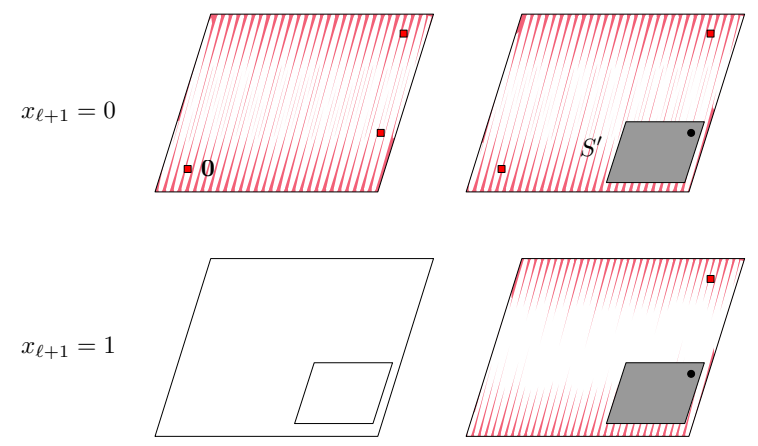

We claim that all the points in $S^{\prime} \triangle e_{\ell} \triangle e_{\ell+1}$ are infeasible. Suppose for a contradiction that, for some $x \in S^{\prime}$, $x \triangle e_{\ell} \triangle e_{\ell+1} \in S$. Recall that $S^{\prime} \subseteq\left\{x: x_{1}=0, x_{2}=1\right\}$. For $i \in\{1,2\}$, consider the 3-dimensional cube $H_{i} \subseteq\{0,1\}^{3}$ containing $x \triangle e_{\ell}, x \triangle e_{\ell+1}, x \triangle e_{i}$. Notice that for $i \in\{1,2\}$,

$$
\left\{x, x \triangle e_{\ell+1}, x \triangle e_{\ell} \triangle e_{\ell+1}\right\} \subseteq S \quad \text { and } \quad\left\{x \triangle e_{i}, x \triangle e_{\ell}, x \triangle e_{i} \triangle e_{\ell}, x \triangle e_{i} \triangle e_{\ell+1}\right\} \subseteq \bar{S} .
$$

Thus, since $H_{1}, H_{2}$ are not fragile by Theorem 1.8 (ii), it follows that $x \triangle e_{1} \triangle e_{\ell} \triangle e_{\ell+1}, x \triangle e_{2} \triangle e_{\ell} \triangle e_{\ell+1} \in S$. To summarize, setting $y:=x \triangle e_{\ell+1},\left\{y \triangle e_{\ell}, y \triangle e_{1} \triangle e_{\ell}, y \triangle e_{2} \triangle e_{\ell}, y\right\} \subseteq S$. Moreover, $\left\{y \triangle e_{1}, y \triangle e_{2}, y \triangle e_{1} \triangle e_{2}\right\}$ $\subseteq \bar{S}$. As a result, since $S$ does not contain antipodal points and the points in $\left\{x: x_{\ell+2}=\cdots=x_{n}=0\right\}$ satisfy $(\diamond)$, it follows that the 3-dimensional restriction of $S$ containing $\left\{y \triangle e_{1}, y \triangle e_{2}, y \triangle e_{\ell}\right\} \triangle \mathbf{1}$ is fragile, a contradiction to Theorem 1.8 (ii). Thus, all the points in $S^{\prime} \triangle e_{\ell} \triangle e_{\ell+1}$ are infeasible.

We next claim that

$$
S \cap\left\{x: x_{\ell+2}=\cdots=x_{n}=0\right\}=S^{\prime} \cup\left(S^{\prime} \triangle e_{\ell+1}\right) .
$$

Suppose otherwise. Pick the closest pair of feasible points $a, b$ such that $a \in S^{\prime} \triangle e_{\ell+1}$ and $b \in\left\{x: x_{\ell+2}=\right.$ $\left.\cdots=x_{n}=0\right\}-\left[S^{\prime} \cup\left(S^{\prime} \triangle e_{\ell+1}\right)\right]$. Since all the points in $S^{\prime} \triangle e_{\ell} \triangle e_{\ell+1}$ are infeasible, it follows that $\operatorname{dist}(a, b) \geq$ 
2. Consider now the restriction of $S$ containing $a, b \triangle e_{n}$ as antipodal points; because all the points in $\{x$ : $\left.x_{\ell+2}=\cdots=x_{n}=0\right\}$ satisfy $(\diamond)$, this restriction is one of $\left\{R_{k, 1}: 1 \leq k \leq \ell-1\right\}$, a contradiction. Thus, $S \cap\left\{x: x_{\ell+2}=\cdots=x_{n}=0\right\}=S^{\prime} \cup\left(S^{\prime} \triangle e_{\ell+1}\right)$, implying in particular that the feasible points in $\left\{x: x_{\ell+2}=\cdots=x_{n}=0\right\}$ form a hypercube, thereby completing the induction step.

(iv) After possibly relabeling coordinates $1,2,3$ and rerouting $K$ according to (1), we may assume that $S^{\prime}=\left\{e_{3}, e_{2}+e_{3}\right\}$ while $K$ remains as $(\mathbf{0}: 1, \ldots, n-1)$. By the Straight Circuit Lemma, $\bar{S} \cap\left\{x: x_{4}=\cdots=\right.$ $\left.x_{n-1}=0, x_{n}=1\right\}=\left\{e_{3}+e_{n}, e_{2}+e_{3}+e_{n}\right\}$.

Consider the straight circuit

$$
K_{1}:=\left(e_{2}: 1,3,4,5, \ldots, n-1,2\right) .
$$

By (2), $K_{1}$ is infeasible and $K_{1} \triangle e_{n}$ is feasible. The induction hypothesis applied to $K_{1}$ implies that the feasible points in $\left\{x: x_{5}=\cdots=x_{n}=0, x_{2}=1\right\}$ form a hypercube, implying in turn that $\left\{e_{2}+e_{4}, e_{1}+e_{2}+e_{4}\right\} \subseteq \bar{S}$, and so by (2), $\left\{e_{2}+e_{4}+e_{n}, e_{1}+e_{2}+e_{4}+e_{n}\right\} \subseteq S$ :
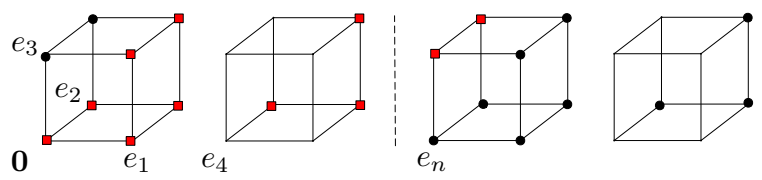

We claim that $e_{1}+e_{3}+e_{4} \in \bar{S}$. Suppose for a contradiction that $e_{1}+e_{3}+e_{4} \in S$. By (2), $e_{1}+e_{3}+e_{4}+e_{n} \in \bar{S}$. By the Sight Propagation Lemma, $e_{1}+e_{4} \in S$, and so by (2), $e_{1}+e_{4}+e_{n} \in \bar{S}$ :
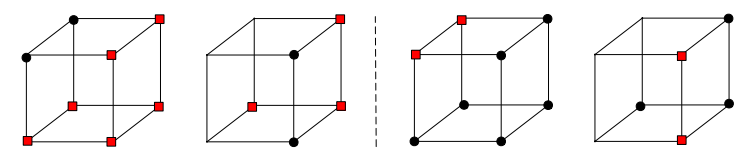

Consider the 3-dimensional restriction of $S$ containing $e_{3}+e_{1}, e_{3}+e_{4}, e_{3}+e_{n}$; as this restriction is neither $P_{3}$ nor $R_{1,1}$, it follows that $e_{3}+e_{4} \in S$. If $e_{4} \in S$, then as $S$ does not have antipodal points and $\mathbf{0}, e_{1}, e_{1}+e_{3}$ satisfy $(\diamond)$ by (2), the 3 -dimensional restriction of $S$ containing $\left\{e_{1}, e_{3}, e_{4}\right\} \triangle \mathbf{1}$ is fragile, thereby contradicting Theorem 1.8 (ii). Otherwise, $e_{4} \notin \bar{S}$. By the Sight Propagation Lemma, $e_{2}+e_{3}+e_{4} \in S$. Consider the straight circuit

$$
K_{2}:=(\mathbf{0}: 4,2,1,3,5, \ldots, n-1) .
$$

By (2), $K_{2}$ is infeasible and $K_{2} \triangle e_{n}$ is feasible. However, the 3-dimensional restriction of $S$ containing $e_{4}+$ $e_{1}, e_{4}+e_{2}, e_{4}+e_{3}$ is a $D_{3}$ whose infeasible points all belong to $K_{3}$, so by Proposition 5.3, $S$ has an $R_{1,1}, R_{5}$ restriction, a contradiction. Thus, $e_{1}+e_{3}+e_{4} \in \bar{S}$, and so by (2), $e_{1}+e_{3}+e_{4}+e_{n} \in S$.

Consider the straight circuit

$$
K_{3}:=(\mathbf{0}: 1,3,4,2,5, \ldots, n-1) .
$$

By (2), $K_{3}$ is infeasible and $K_{3} \triangle e_{n}$ is feasible. The induction hypothesis applied to $K_{3}$ tells us that the feasible points in $\left\{x: x_{5}=\cdots=x_{n}=0, x_{2}=0\right\}$ form a hypercube, implying in turn that $\left\{e_{4}, e_{1}+e_{4}\right\} \subseteq \bar{S}$. By (2), $\left\{e_{4}+e_{n}, e_{1}+e_{4}+e_{n}\right\} \subseteq S$ : 

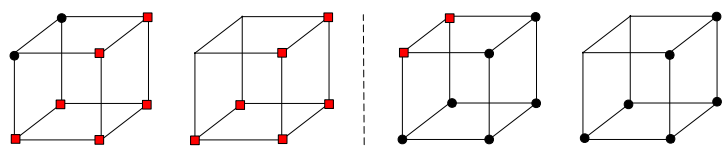

Resistance now implies that the feasible points in $\left\{x: x_{5}=\cdots=x_{n}=0\right\}$ form a hypercube, thereby completing the induction step.

(v) After possibly relabeling coordinates $1, \ldots, \ell$ and rerouting $K$ according to (1), we may assume that $S^{\prime}=\left\{x: x_{\ell-1}=0, x_{\ell}=1, x_{\ell+2}=\cdots=x_{n}=0\right\}$ while $K$ remains as $(0: 1, \ldots, n-1)$. As $\ell-2 \geq 2$, the points in $S^{\prime}$ are active in directions 1,2. Let us apply the induction hypothesis to the straight infeasible circuit $K$ but with a different starting point $\left(e_{1}: 2, \ldots, n-1,1\right)$, given that the points in $\left\{x: x_{\ell+1}=\cdots=x_{n}=0, x_{1}=\right.$ $1\}$ satisfy $(\diamond)$ and its feasible points $S^{\prime} \cap\left\{x: x_{1}=1\right\}$ form a hypercube; and to the straight infeasible circuit $K_{4}:=\left(e_{2}: 1,3, \ldots, n-1,2\right)$ satisfying $V\left(K_{4} \triangle e_{n}\right) \subseteq S$, given that the points in $\left\{x: x_{\ell+1}=\cdots=x_{n}=\right.$ $\left.0, x_{2}=1\right\}$ satisfy $(\diamond)$ and its feasible points $S^{\prime} \cap\left\{x: x_{2}=1\right\}$ form a hypercube. The induction hypothesis implies that

(5) the points in $\left\{x: x_{\ell+2}=\cdots=x_{n}=0, x_{1}=1\right\}$ satisfy $(\diamond)$ and its feasible points form a hypercube,

and that the points in $\left\{x: x_{\ell+2}=\cdots=x_{n}=0, x_{2}=1\right\}$ satisfy $(\diamond)$ and its feasible points form a hypercube. The latter implies in particular that $\sum_{i=1}^{\ell+1} e_{i}-e_{1} \in \bar{S}$. We will next apply the induction hypothesis to the straight infeasible circuit $K_{5}:=(\mathbf{0}: 2, \ldots, \ell+1,1, \ell+1, \ldots, n-1)$ satisfying $V\left(K_{5} \triangle e_{n}\right) \subseteq S$, given that the points in $\left\{x: x_{\ell+1}=\cdots=x_{n}=0, x_{1}=0\right\}$ satisfy $(\diamond)$ and its feasible points $S^{\prime} \cap\left\{x: x_{1}=0\right\}$ form a hypercube. The induction hypothesis tells us that

(6) the points in $\left\{x: x_{\ell+2}=\cdots=x_{n}=0, x_{1}=0\right\}$ satisfy $(\diamond)$ and its feasible points form a hypercube.

By (5) and (6), the points in $\left\{x: x_{\ell+2}=\cdots=x_{n}=0\right\}$ satisfy $(\diamond)$ and the feasible points of $\left\{x: x_{\ell+2}=\cdots=\right.$ $\left.x_{n}=0\right\}$ are contained in $S^{\prime} \cup\left(S^{\prime} \triangle e_{\ell+1}\right)$ :

$$
x_{\ell}=0 \quad x_{\ell}=1
$$

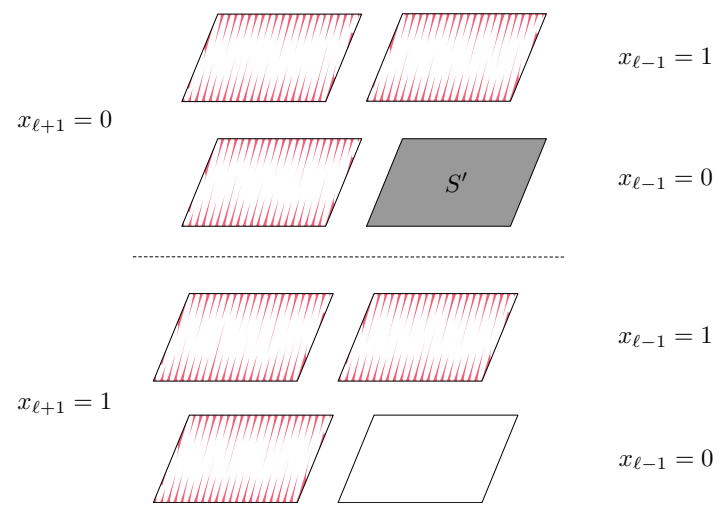


After applying the Plane Propagation Lemma to the 0-restriction of $S$ at coordinates $\ell+2, \ldots, n$, we see that the feasible points in $\left\{x: x_{\ell+2}=\cdots=x_{n}=0\right\}$ must in fact form a hypercube, thereby completing the induction step. This finishes the proof of the lemma.

We are now ready to prove the main result of this section:

Theorem 5.5. Take an integer $n \geq 4$ and a resistant set $S \subseteq\{0,1\}^{n}$ that is non-polar. Assume that there is a straight infeasible circuit $K$ of length $2(n-1)$ contained in $\left\{x: x_{n}=0\right\}$ such that $V\left(K \triangle e_{n}\right) \subseteq S$. Then $S$ has one of $\left\{R_{k, 1}: 1 \leq k \leq n-2\right\} \cup\left\{R_{5}\right\}$ as a restriction.

Proof. After a possible twisting and relabeling, we may assume that $K=(\mathbf{0}: 1,2, \ldots, n-1)$.

Claim. If $n=4$, then $S \cong R_{2,1}$.

Proof of Claim. Suppose that $n=4$. As $V(K) \subseteq \bar{S}$ and $V\left(K \triangle e_{4}\right) \subseteq S$, it follows that

$$
\{0000,1000,1100,1110,0110,0010\} \subseteq \bar{S} \quad \text { and } \quad\{0001,1001,1101,1111,0111,0011\} \subseteq S .
$$

Since $S$ is non-polar, $|\{1010,0100\} \cap S| \geq 1$. Since 1010,0100 are both adjacent to a vertex of $K$, it follows from the Straight Circuit Lemma that $\{1010,0100\} \subseteq S$ and $\{0101,1011\} \subseteq \bar{S}$, implying in turn that $S \cong R_{2,1}$, as required.

We may therefore assume that $n \geq 5$. By the Straight Circuit Lemma, the points of $\left\{x: x_{3}=\cdots=x_{n}=0\right\}$ satisfy $(\diamond)$. Also, as $\left\{x: x_{3}=\cdots=x_{n}=0\right\}$ contains at most one feasible point, the hypotheses of Lemma 5.4 hold for $\ell=2$. If $S$ has one of $\left\{R_{k, 1}: 1 \leq k \leq n-3\right\} \cup\left\{R_{5}\right\}$ as a restriction, then we are done. Otherwise, after applying Lemma 5.4 for $\ell=2, \ldots, n-3$ in this order, we see that the points in $\left\{x: x_{n-1}=x_{n}=0\right\}$ satisfy $(\diamond)$, implying in turn that all the points in $\{0,1\}^{n}$ satisfy $(\diamond)$, and that $S^{\prime}:=S \cap\left\{x: x_{n-1}=x_{n}=0\right\}$ is a hypercube. Since $S$ is non-polar and $(\diamond)$ holds, it follows that $S^{\prime} \neq \emptyset$. Pick a closest pair of feasible points $a, b$ such that $a \in S^{\prime}$ and $b \in\left(S \cap\left\{x: x_{n}=0\right\}\right)-S^{\prime}=S^{\prime} \triangle \mathbf{1} \triangle e_{n}$. Notice that $\operatorname{dist}(a, b) \geq 2$. It follows from $(\diamond)$ that the restriction of $S$ containing $a, b \triangle e_{n}$ as antipodal points is one of $\left\{R_{k, 1}: 1 \leq k \leq n-2\right\}$. In either one of the two cases, $S$ has one of $\left\{R_{k, 1}: 1 \leq k \leq n-2\right\} \cup\left\{R_{5}\right\}$ as a restriction, as required.

\section{Proofs of Theorems 1.17 and 1.18}

Let us start with the following result:

Proposition 6.1. Take an integer $n \geq 3$ and a resistant set $S \subseteq\{0,1\}^{n}$ without antipodal points. If every straight infeasible path has length at most $n-2$, then $S$ has an $R_{1,1}$ restriction.

Proof. Let $m \leq n-2$ be the maximum length of a straight infeasible path. Then every straight infeasible path has length at most $m$. As $S$ does not have antipodal points, it follows that

$(\star)$ every straight feasible path has length at most $m$, 
because the antipode of every straight feasible path is a straight infeasible path of the same length. Let $P:=$ $\left(v_{0}, v_{1}, \ldots, v_{m}\right)$ be a maximum length straight infeasible path. After a possible twisting and relabeling, we may assume that $v_{0}=\mathbf{0}$ and $v_{j}=v_{j-1} \triangle e_{j}$ for $j \in[m]$. Our maximal choice of $P$ implies that for each $j \in\{m+1, \ldots, n\}$, the points $v_{0} \triangle e_{j}, v_{m} \triangle e_{j}$ are feasible. Thus, by the Path Propagation Lemma,

$(\diamond)$ for each $j \in\{m+1, \ldots, n\}, P \triangle e_{j}$ is a feasible path.

If $m=n-2$, then $v_{0} \triangle e_{m+1}=e_{n-1}$ and $v_{m} \triangle e_{m+2}=\mathbf{1}-e_{n-1}$ are feasible points by $(\diamond)$, which cannot be the case as there are no antipodal feasible points. Thus, $m \leq n-3$. Let

$$
R:=S \cap\left\{x: x_{i}=0, i \notin\{m+1, m+2, m+3\}\right\} .
$$

By assumption, $\mathbf{0} \notin R$, and by $(\diamond), e_{m+1}, e_{m+2}, e_{m+3} \in R$. Moreover, by $(\star), P \triangle e_{m+1}, P \triangle e_{m+2}, P \triangle e_{m+3}$ are maximal straight feasible paths, so $e_{m+1} \triangle e_{m+2}, e_{m+2} \triangle e_{m+3}, e_{m+3} \triangle e_{m+1} \notin R$. As $S$ is resistant, it does not have a fragile restriction by Theorem 1.8 (ii), so $e_{m+1} \triangle e_{m+2} \triangle e_{m+3} \in R$. As a result, after dropping coordinates $[n]-\{m+1, m+2, m+3\}$ from $R$ we obtain an $R_{1,1}$, so $S$ has an $R_{1,1}$ restriction, as required.

Using Theorem 5.5 and Proposition 6.1, we prove the following:

Theorem 6.2. Take an integer $n \geq 3$ and a resistant set $S \subseteq\{0,1\}^{n}$ that is non-polar. If every straight infeasible path has length at most $n-1$, then $S$ has one of $\left\{R_{k, 1}: k \geq 1\right\} \cup\left\{R_{5}\right\}$ as a restriction.

Proof. If there is no straight infeasible path of length $n-1$, then $S$ has an $R_{1,1}$ restriction by Proposition 6.1, so we are done. Otherwise, there is a straight infeasible path $P:=\left(v_{0}, v_{1}, \ldots, v_{n-1}\right)$ of length $n-1$, which by assumption is maximal. After a possible relabeling and twisting, if necessary, we may assume that $V(P) \subseteq$ $\left\{x: x_{n}=0\right\}$. Maximality of $P$ implies that $v_{0} \triangle e_{n}, v_{n-1} \triangle e_{n}$ are feasible, so by the Path Propagation Lemma, $P \triangle e_{n}$ is a feasible path. As $S$ does not contain antipodal points, it follows that the path $Q:=P \triangle e_{n} \triangle \mathbf{1}$ is infeasible. Since $Q$ is a straight infeasible $\left(v_{n-1}, v_{0}\right)$-path, and $v_{n-1} \triangle e_{n}, v_{0} \triangle e_{n} \in S$, we get from the Path Propagation Lemma that $Q \triangle e_{n}$ is a feasible path. Consider the straight infeasible circuit $K:=P \cup Q$ of length $2(n-1)$ contained in $\left\{x: x_{n}=0\right\}$. We just showed that $V\left(K \triangle e_{n}\right) \subseteq S$. Thus, by Theorem 5.5, $S$ has one of $\left\{R_{k, 1}: k \geq 1\right\} \cup\left\{R_{5}\right\}$ as a restriction, as required.

We are now ready to prove Theorem 1.17, stating that up to isomorphism, $\left\{R_{k, 1}: k \geq 1\right\} \cup\left\{R_{5}\right\}$ are the only half-dense strictly non-polar sets that are resistant:

Proof of Theorem 1.17. Take an integer $n \geq 3$ and a half-dense strictly non-polar set $S \subseteq\{0,1\}^{n}$ that is resistant. Since $S$ is non-polar and half-dense, it follows that for each $x \in\{0,1\}^{n}$, one of $x, \mathbf{1}-x$ is feasible and the other is infeasible. In particular, there is no antipodal pair of infeasible points. Since a straight path of length $n$ has antipodal points as ends, it therefore follows that every straight infeasible path has length at most $n-1$. Hence, as $S$ is resistant and non-polar, Theorem 6.2 implies that $S$ has one of $\left\{R_{k, 1}: k \geq 1\right\} \cup\left\{R_{5}\right\}$ as a restriction. As $\left\{R_{k, 1}: k \geq 1\right\} \cup\left\{R_{5}\right\}$ are non-polar, and $S$ is strictly non-polar, $S$ must be isomorphic to one of $\left\{R_{k, 1}: k \geq 1\right\} \cup\left\{R_{5}\right\}$, as required. 
Next we prove Theorem 1.18, which states the following:

Take integers $n_{1}, n_{2} \geq 1$ and sets $S_{1} \subseteq\{0,1\}^{n_{1}}, S_{2} \subseteq\{0,1\}^{n_{2}}$, where $S_{1}, \overline{S_{1}}, S_{2}, \overline{S_{2}}$ are nonempty and resistant. Then $S_{1} * S_{2}$ is strictly polar if, and only if, $S_{1} * S_{2}$ has none of $\left\{R_{k, 1}: k \geq 1\right\} \cup\left\{R_{5}\right\}$ as a restriction.

This theorem is by and large a consequence of Corollary 4.15 and Theorem 1.17. The proof also relies on the following result:

Proposition 6.3 ([3], Proposition 5.11). Take integers $n_{1}, n_{2} \geq 1$ and sets $S_{1} \subseteq\{0,1\}^{n_{1}}, S_{2} \subseteq\{0,1\}^{n_{2}}$, where $S_{1}, \overline{S_{1}}, S_{2}, \overline{S_{2}}$ are nonempty. If one of $S_{1}, \overline{S_{1}}, S_{2}, \overline{S_{2}}$ is not strictly connected, then $S_{1} * S_{2}$ has one of $\left\{R_{k, 1}: k \geq 1\right\}$ as a restriction.

We will also need the following result:

Theorem 6.4 ([3], Theorem 1.18 (2)). Take integers $n_{1}, n_{2} \geq 1$ and sets $S_{1} \subseteq\{0,1\}^{n_{1}}, S_{2} \subseteq\{0,1\}^{n_{2}}$, where $S_{1} * S_{2}$ is strictly non-polar. Then either $n_{1}=1$ or $n_{2}=1$. In particular, $S_{1} * S_{2}$ is half-dense.

We are now ready to prove the final result of the paper, Theorem 1.18:

Proof of Theorem 1.18. $(\Rightarrow)$ holds trivially. $(\Leftarrow)$ Assume that $S_{1} * S_{2}$ has a non-polar restriction. We need to show that $S_{1} * S_{2}$ has one of $\left\{R_{k, 1}: k \geq 1\right\} \cup\left\{R_{5}\right\}$ as a restriction. If one of $S_{1}, \overline{S_{1}}, S_{2}, \overline{S_{2}}$ is not strictly connected, then by Proposition 6.3, $S_{1} * S_{2}$ has one of $\left\{R_{k, 1}: k \geq 1\right\}$ as a restriction, so we are done. Otherwise, $S_{1}, \overline{S_{1}}, S_{2}, \overline{S_{2}}$ are strictly connected. Since they are also resistant, Corollary 4.15 implies that $S_{1}, \overline{S_{1}}, S_{2}, \overline{S_{2}}$ are strictly polar.

For each $i \in\{1,2\}$, take an integer $m_{i} \geq 0$ and a restriction $R_{i} \subseteq\{0,1\}^{m_{i}}$ so that $R_{1} * R_{2}$ is a strictly non-polar restriction of $S_{1} * S_{2}$. As restrictions of $S_{1}$ and $S_{2}, R_{1}$ and $R_{2}$ are both polar, implying in turn that $m_{1} \geq 1$ and $m_{2} \geq 1$. Therefore, $R_{1} * R_{2}$ is half-dense by Theorem 6.4. As restrictions of $S_{1}, \overline{S_{1}}, S_{2}, \overline{S_{2}}$, the sets $R_{1}, \bar{R}_{1}, R_{2}, \overline{R_{2}}$ are resistant by Remark 3.2. Thus, $R_{1} * R_{2}$ is resistant by Theorem 1.7 (3). As a result, $R_{1} * R_{2}$ is isomorphic to one of $\left\{R_{k, 1}: k \geq 1\right\} \cup\left\{R_{5}\right\}$ by Theorem 1.17, in turn finishing the proof.

\section{The max-flow min-cut property and Theorem 1.13}

Let $\mathcal{C}$ be a clutter over ground set $E$. A cover is a subset of $E$ that intersects every member. Take weights $w \in \mathbb{Z}_{+}^{E}$. A w-weighted packing is a collection of (possibly equal) members such that every element $e$ appears in at most $w_{e}$ members; its value is the number of members in the collection. Given a cover $B$ and a $w$-weighted packing $C_{1}, \ldots, C_{k}$, we have that

$$
w(B)=\sum_{e \in B} w_{e} \geq \sum_{e \in B}\left|\left\{i \in[k]: e \in C_{i}\right\}\right|=\sum_{i \in[k]}\left|B \cap C_{i}\right| \geq k .
$$

That is, the weight of every cover is at least as large as the value of every $w$-weighted packing. Denote by $\tau(\mathcal{C}, w)$ the minimum weight of a cover, and by $\nu(\mathcal{C}, w)$ the maximum value of a $w$-weighted packing. Then 
$\tau(\mathcal{C}, w) \geq \nu(\mathcal{C}, w)$. Observe that $\mathcal{C}$ packs if, and only if, $\tau(\mathcal{C}, \mathbf{1})=\nu(\mathcal{C}, \mathbf{1})$. Moreover, $\mathcal{C}$ has the packing property if, and only if,

$$
\tau(\mathcal{C}, w)=\nu(\mathcal{C}, w) \quad \forall w \in\{0,1, \infty\}^{E} .
$$

We say that $\mathcal{C}$ has the max-flow min-cut property if

$$
\tau(\mathcal{C}, w)=\nu(\mathcal{C}, w) \quad \forall w \in \mathbb{Z}_{+}^{E}
$$

Clearly, the max-flow min-cut property implies the packing property. The Replication Conjecture of Conforti and Cornuéjols [9] predicts the converse should also hold, that

(?) the packing property implies the max-flow min-cut property. (?)

Corollary 1.12 showed that if a set is resistant and strictly polar, then its cuboid has the packing property; if the Replication Conjecture were true, then the cuboid should also have the max-flow min-cut property. This is what Theorem 1.13 proves, without relying on the Replication Conjecture. We will need the following proposition:

Proposition 7.1. Take an integer $n \geq 1$, a polar set $S \subseteq\{0,1\}^{n}$, and weights $w \in \mathbb{Z}_{+}^{2 n}$ such that

(hl) $\tau(\operatorname{cuboid}(S), w)>\nu(\operatorname{cuboid}(S), w)$,

(h2) for any $w^{\prime} \in \mathbb{Z}_{+}^{2 n}$ such that $\sum_{e \in[2 n]} w_{e}^{\prime}<\sum_{e \in[2 n]} w_{e}$, we have that

$$
\tau\left(\operatorname{cuboid}(S), w^{\prime}\right)=\nu\left(\operatorname{cuboid}(S), w^{\prime}\right),
$$

(h3) the cuboid of every proper restriction of $S$ has the max-flow min-cut property.

Given that $\tau:=\tau(\operatorname{cuboid}(S), w)$, the following statements hold:

(c1) for each $i \in[n],\{2 i-1,2 i\}$ is a minimum weight cover,

(c2) for each element e, $\tau-1 \geq w_{e} \geq 1$,

(c3) $\tau \geq 3$, and

(c4) for every member $C$ of cuboid $(S)$, there is a minimal cover $B$ such that

$$
w(B) \leq \tau-2+|B \cap C|
$$

Moreover, for every such B,

(c5) B has at most two elements of weight at least $\frac{\tau}{2}$, and

(c6) if $B$ has two elements $f, g$ of weight at least $\frac{\tau}{2}$, then $B \subseteq C, w_{f}=w_{g}=\frac{\tau}{2}$, and $w_{e}=1$ for all $e \in B-\{f, g\}$.

Proof. (c1) Let us first prove that 
every element $e$ of $\operatorname{cuboid}(S)$ appears in a minimum weight cover.

Suppose otherwise. In particular, $w_{e} \geq 1$. Let $w^{\prime}$ be obtained from $w$ after decreasing the weight of $e$ by 1 . Our contrary assumption implies that $\tau\left(\operatorname{cuboid}(S), w^{\prime}\right)=\tau$. It follows from (h2) that $\tau=\tau\left(\operatorname{cuboid}(S), w^{\prime}\right)=$ $\nu\left(\operatorname{cuboid}(S), w^{\prime}\right)$, so there is a $w^{\prime}$-weighted packing of value $\tau$, which is also a $w$-weighted packing of value $\tau$, a contradiction to (h1).

Pick minimum weight covers $B_{1}, B_{2}$ containing $2 i-1,2 i$, respectively. If $\{2 i-1,2 i\} \subseteq B_{1}$ or $\{2 i-1,2 i\} \subseteq$ $B_{2}$, then $\{2 i-1,2 i\}$ is also a minimum weight cover, so we are done. Otherwise, $B_{1} \cap\{2 i-1,2 i\}=\{2 i-1\}$ and $B_{2} \cap\{2 i-1,2 i\}=\{2 i\}$. As a result, $\left(B_{1} \cup B_{2}\right)-\{2 i-1,2 i\}$ is a cover, and so its weight is at least $\tau$. Since $\{2 i-1,2 i\}$ is a cover as well, its weight is also at least $\tau$, so

$$
2 \tau=w\left(B_{1}\right)+w\left(B_{2}\right) \geq w\left(\left(B_{1} \cup B_{2}\right)-\{2 i-1,2 i\}\right)+w(\{2 i-1,2 i\}) \geq 2 \tau,
$$

and so equality holds throughout. Subsequently, $\{2 i-1,2 i\}$ is a minimum weight cover, as required.

(c2) Take $i \in[n]$. By (c1), $w_{2 i-1}+w_{2 i}=\tau$. It therefore suffices to show that $\left\{w_{2 i-1}, w_{2 i}\right\} \neq\{0, \tau\}$. Suppose otherwise. After a possible twisting and relabeling, we may assume that $i=n, w_{2 n-1}=0$ and $w_{2 n}=\tau$. Define $w^{\prime} \in \mathbb{Z}_{+}^{2 n-2}$ as follows:

$$
w_{e}^{\prime}:=w_{e} \quad \forall e \in[2 n-2] .
$$

Let $S^{\prime} \subseteq\{0,1\}^{n-1}$ be the 0 -restriction of $S$ at coordinate $n$. By (h3), cuboid $\left(S^{\prime}\right)$ has the max-flow min-cut property, so

$$
\tau\left(\operatorname{cuboid}\left(S^{\prime}\right), w^{\prime}\right)=\nu\left(\operatorname{cuboid}\left(S^{\prime}\right), w^{\prime}\right)
$$

Notice however that $\operatorname{cuboid}\left(S^{\prime}\right)=\operatorname{cuboid}(S) \backslash 2 i-1 / 2 i$. Thus, since $w_{2 n-1}=0$,

$$
\tau\left(\operatorname{cuboid}\left(S^{\prime}\right), w^{\prime}\right)=\tau(\operatorname{cuboid}(S), w)=\tau .
$$

So cuboid $\left(S^{\prime}\right)$ has a $w^{\prime}$-weighted packing of value $\tau$, and as $w_{2 n}=\tau$, we get a $w$-weighted packing of value $\tau$ in $\operatorname{cuboid}(S)$, a contradiction to (h1).

(c3) Clearly, $\tau \geq 2$. Suppose for a contradiction that $\tau=2$. Then by (c2), every element must have weight 1 . However, as $S$ is polar, $\tau(\operatorname{cuboid}(S), \mathbf{1})=\nu(\operatorname{cuboid}(S), \mathbf{1})$, a contradiction to (h1).

(c4) Suppose for a contradiction that for every minimal cover $B$,

$$
w(B)-|B \cap C| \geq \tau-1 .
$$

Let $w^{\prime} \in \mathbb{R}_{+}^{2 n}$ be obtained from $w$ after decreasing the weight of every element in $C$ by 1 . The inequality above implies that $\tau\left(\operatorname{cuboid}(S), w^{\prime}\right) \geq \tau-1$. It follows from (h2) that $\nu\left(\operatorname{cuboid}(S), w^{\prime}\right)=\tau\left(\operatorname{cuboid}(S), w^{\prime}\right) \geq \tau-1$. As a result, $\operatorname{cuboid}(S)$ has a $w^{\prime}$-weighted packing of value $\tau-1$, which together with $C$ yields a $w$-weighted packing of value $\tau$, a contradiction to (h1). (c5) Suppose for a contradiction that there are three elements $e, f, g \in B$ of weight at least $\frac{\tau}{2}$. Since every other element has weight at least 1 by (c2),

$$
\tau-2+|B| \geq \tau-2+|B \cap C| \geq w(B) \geq\left(3 \times \frac{\tau}{2}\right)+|B|-3
$$


implying in turn that $2 \geq \tau$, a contradiction to (c3). (c6) Assume that $B$ has elements $f, g$ of weight at least $\frac{\tau}{2}$. Since every other element has weight at least 1 by (c2),

$$
\tau-2+|B| \geq \tau-2+|B \cap C| \geq w(B)=w_{f}+w_{g}+\sum_{e \in B-\{f, g\}} w_{e} \geq \frac{\tau}{2}+\frac{\tau}{2}+|B|-2 .
$$

As a result, equality holds throughout, implying in turn that (c6) holds.

We will also need the following remark about resistant sets:

Remark 7.2. Take an integer $n \geq 1$ and a resistant set $S \subseteq\{0,1\}^{n}$, where 0 is infeasible. Let $p^{1}, \ldots, p^{k}$ be the feasible points of minimal support, and let $C_{1}, \ldots, C_{k}$ be the corresponding members of cuboid $(S)$. Then

- $C_{1} \cap\{1,3, \ldots, 2 n-1\}, \ldots, C_{k} \cap\{1,3, \ldots, 2 n-1\}$ are pairwise disjoint,

- for every subset $B \subseteq\{1,3, \ldots, 2 n-1\}, B$ is a cover of $\operatorname{cuboid}(S)$ if, and only if,

$$
B \cap C_{j} \neq \emptyset \quad \forall j \in[k] .
$$

In particular, $B$ is a minimal cover of cuboid $(S)$ if, and only if,

$$
\left|B \cap C_{j}\right|=1 \quad \forall j \in[k] .
$$

Proof. Since $S$ is resistant, $p^{1}, \ldots, p^{k}$ have pairwise disjoint supports, implying in turn that $C_{1} \cap\{1,3, \ldots, 2 n-$ $1\}, \ldots, C_{k} \cap\{1,3, \ldots, 2 n-1\}$ are pairwise disjoint. For every member $C$ of $\operatorname{cuboid}(S), C \cap\{2 i-1: i \in[n]\}$ contains one of $C_{j} \cap\{2 i-1: i \in[n]\}, j \in[k]$. Put together, these facts imply that for every subset $B \subseteq$ $\{1,3, \ldots, 2 n-1\}, B$ is a cover of $\operatorname{cuboid}(S)$ if, and only if,

$$
B \cap C_{j} \neq \emptyset \quad \forall j \in[k],
$$

as required.

Lastly, we will need the following remark:

Remark 7.3 ([28]). If a clutter is ideal, then so is every minor of it.

We are now ready to prove Theorem 1.13, stating that every resistant, strictly polar set has a cuboid with the max-flow min-cut property:

Proof of Theorem 1.13. Take an integer $n \geq 1$ and a resistant, strictly polar set $S \subseteq\{0,1\}^{n}$. By Remark 3.2, every restriction of $S$ is resistant. Since every restriction of $S$ is strictly polar as well, we may assume that the cuboid of every proper restriction of $S$ has the max-flow min-cut property. Suppose for a contradiction that $S$ does not have the max-flow min-cut property. Choose weights $w \in \mathbb{Z}_{+}^{2 n}$ such that

$$
\tau(\operatorname{cuboid}(S), w)>\nu(\operatorname{cuboid}(S), w)
$$


and subject to satisfying this inequality, $\sum_{e \in[2 n]} w_{e}$ is minimized. By Proposition 7.1, as hypotheses (h1)-(h3) hold, consequences (c1)-(c6) follow. In particular, setting $\tau:=\tau(\operatorname{cuboid}(S), w)$, we have by (c1) that

$$
w_{2 i-1}+w_{2 i}=\tau \quad \forall i \in[n] .
$$

Going forward, given $B \subseteq\{1,2, \ldots, 2 n-1,2 n\}$, define

$$
B^{\text {odd }}:=B \cap\{1,3, \ldots, 2 n-1\} \quad B^{\text {even }}:=B \cap\{2,4, \ldots, 2 n\} .
$$

Claim 1. Assume that $w_{2 i} \geq \frac{\tau}{2} \geq w_{2 i-1}$ for each $i \in[n]$, and $\mathbf{0}$ is infeasible. Let $p^{1}, \ldots, p^{k}$ be the feasible points of minimal support, for some integer $k \geq 1$, and let $C_{1}, \ldots, C_{k}$ be the corresponding members of cuboid $(S)$. Then the following statements hold:

(1) $k \geq 2$,

(2) if $B^{\text {even }}=\{2 n\}$ and $B$ is a minimal cover not containing $2 n-1$, then

$$
\left|\left\{j \in[k]: B \cap C_{j}^{\text {odd }}=\emptyset\right\}\right|=1 .
$$

Moreover,

(3) for each $j \in[k], w(B) \geq \tau-2+\left|B \cap C_{j}\right|$,

Given that $B \cap C_{k}^{\text {odd }}=\emptyset$ and $w(B)=\tau-2+\left|B \cap C_{1}\right|$, then

(4) for each $c \in C_{k}^{\text {odd }}$, we have that $w_{c}=\frac{\tau}{2}$, and

(5) for each $i \in[k-1]$ and $c_{i} \in B \cap C_{i}^{o d d}$, we have that $\sum_{i=1}^{k-1} w_{c_{i}}=\frac{\tau}{2}$.

(6) if $B^{\text {even }}=\{2 n-2,2 n\}$ and $B$ is a minimal cover not containing either of $2 n-3,2 n-1$, then either $e_{n-1}, e_{n}$ are both feasible, or

$$
\left|\left\{j \in[k]: B \cap C_{j}^{\text {odd }}=\emptyset\right\}\right|=1 \text { or } 2 .
$$

Proof of Claim. (1) Suppose otherwise. Then $k=1$. Pick an element $c \in C_{1}^{\text {odd }}$. Then $\{c\}$ is a minimal cover by Remark 7.2. However,

$$
w(\{c\})=w_{c} \leq \tau-1
$$

by (c2), a contradiction as every cover has weight at least $\tau$.

(2) As $B-\{2 n\}=B^{\text {odd }}$ is not a cover, it follows from Remark 7.2 that $B^{\text {odd }}$ is disjoint from one of $C_{1}, \ldots, C_{k}$, say $B \cap C_{k}^{\text {odd }}=B^{\text {odd }} \cap C_{k}=\emptyset$. We need to show that

$$
B \cap C_{j}^{\text {odd }} \neq \emptyset \quad \forall j \in[k-1] .
$$

Suppose otherwise; say $B \cap C_{k-1}^{\text {odd }}=\emptyset$. Since $B$ is a cover, it intersects both $C_{k-1}$ and $C_{k}$, so $2 n \in C_{k-1} \cap C_{k}$ and in turn $p_{n}^{k-1}=p_{n}^{k}=0$. Moreover, as the cover $B$ does not contain $2 n-1$, the point $e_{n}$ is infeasible. Consider the valid pairs $\left[\mathbf{0}, p^{k-1}\right],\left[\mathbf{0}, p^{k}\right]$ as well as the valid sequence $(n)$ for $\mathbf{0}$. Let

$$
q^{k-1}:=\operatorname{im}\left[\mathbf{0}, p^{k-1}\right](n) \in\left\{p^{k-1}, p^{k-1} \triangle e_{n}\right\} \quad \text { and } \quad q^{k}:=\operatorname{im}\left[\mathbf{0}, p^{k}\right](n) \in\left\{p^{k}, p^{k} \triangle e_{n}\right\} .
$$


By Remark 4.9 and Theorem 4.11, the points $q^{k-1}, q^{k}$ are distinct, feasible and seen by $e_{n}$. As $S$ is resistant, the points $q^{k-1} \triangle e_{n}, q^{k} \triangle e_{n}$ have disjoint supports. Hence, since $p_{n}^{k-1}=p_{n}^{k}=0$, either $q^{k-1}=p^{k-1} \triangle e_{n}$ or $q^{k}=p^{k} \triangle e_{n}$. In particular, one of $C_{k-1} \triangle\{2 n-1,2 n\}, C_{k} \triangle\{2 n-1,2 n\}$ is a member of cuboid $(S)$. However, since $B \cap C_{k-1}=B \cap C_{k}=\{2 n\}$, both $C_{k-1} \triangle\{2 n-1,2 n\}, C_{k} \triangle\{2 n-1,2 n\}$ are disjoint from the cover $B$, a contradiction. Thus,

$$
\left\{j \in[k]: B \cap C_{j}^{\text {odd }}=\emptyset\right\}=\{k\} .
$$

(3) Since $B \cap C_{k}=\{2 n\}$, the inequality holds (strictly) for $j=k$. It therefore suffices to prove the inequality for $j=1$. By Remark 7.2, $C_{1}^{\text {odd }}, \ldots, C_{k-1}^{\text {odd }}$ are pairwise disjoint, so

$$
\begin{aligned}
w(B) & \geq w_{2 n}+\sum_{i=1}^{k-1} w\left(B \cap C_{i}^{\text {odd }}\right) \\
& \geq w_{2 n}+w\left(\left(B \cap C_{1}^{\text {odd }}\right)-c_{1}\right)+\sum_{i=1}^{k-1} w_{c_{i}} \quad \forall c_{i} \in B \cap C_{i}^{\text {odd }} \\
& \geq w_{2 n}+w\left(\left(B \cap C_{1}^{\text {odd }}\right)-c_{1}\right)+\tau-w_{c_{k}} \quad \forall c_{k} \in C_{k}^{\text {odd }} \\
& \geq\left|\left(B \cap C_{1}^{\text {odd }}\right)-c_{1}\right|+\tau+w_{2 n}-w_{c_{k}} \\
& =\left|B \cap C_{1}^{\text {odd }}\right|-1+\tau+w_{2 n}-w_{c_{k}} \\
& \geq\left|B \cap C_{1}\right|-\left|B^{\text {even }}\right|-1+\tau+w_{2 n}-w_{c_{k}} \\
& =\left|B \cap C_{1}\right|-2+\tau+\left(w_{2 n}-\frac{\tau}{2}\right)+\left(\frac{\tau}{2}-w_{c_{k}}\right) \\
& \geq\left|B \cap C_{1}\right|-2+\tau,
\end{aligned}
$$

where the third inequality follows from the inequality $\sum_{i=1}^{k} w_{c_{i}} \geq \tau$ which holds because $\left\{c_{1}, \ldots, c_{k}\right\}$ is a cover of $\operatorname{cuboid}(S)$ by Remark 7.2, and the last inequality holds because $w_{2 n} \geq \frac{\tau}{2} \geq w_{c_{k}}$ due to our assumption. Thus, $w(B) \geq\left|B \cap C_{1}\right|-2+\tau$. Suppose that equality holds here. Then equality must hold in every line of the inequalities above. (4) follows from the last inequality above holding at equality. (5) Pick $c_{k} \in C_{k}^{\text {odd }}$. Since the third inequality holds at equality, we have that $\sum_{i=1}^{k-1} w_{c_{i}}=\tau-w_{c_{k}}=\frac{\tau}{2}$ by (4), as required.

(6) Assume that one of $e_{n-1}, e_{n}$, say $e_{n-1}$, is infeasible. Since $B$ does not contain either of $2 n-3,2 n-1$, the point $e_{n-1}+e_{n}$ is also infeasible. Thus, the three points $\mathbf{0}, e_{n-1}, e_{n-1}+e_{n}$ are infeasible, and so the sequence $(n-1, n)$ is valid for $\mathbf{0}$.

As $B-\{2 n-2,2 n\}=B^{\text {odd }}$ is not a cover, Remark 7.2 tells us that $B^{\text {odd }}$ is disjoint from one of $C_{1}, \ldots, C_{k}$, say $B \cap C_{k}^{\text {odd }}=B^{\text {odd }} \cap C_{k}=\emptyset$. We need to show that

$$
\left|\left\{j \in[k-1]: B \cap C_{j}^{\text {odd }}=\emptyset\right\}\right| \leq 1
$$

Suppose otherwise; say $B \cap C_{k-2}^{\text {odd }}=B \cap C_{k-1}^{\text {odd }}=\emptyset$. For each $j \in\{k-2, k-1, k\}$, take the valid pair $\left[\mathbf{0}, p^{j}\right]$ and let

$$
q^{j}:=\operatorname{im}\left[\mathbf{0}, p^{j}\right](n-1, n) \in\left\{p^{j}, p^{j} \triangle e_{n-1}, p^{j} \triangle e_{n}, p^{j} \triangle e_{n-1} \triangle e_{n}\right\}
$$


By Remark 4.9 and Theorem 4.11, the points $q^{k-2}, q^{k-1}, q^{k}$ are distinct, feasible and seen by $e_{n-1} \triangle e_{n}$. Thus, as $S$ is resistant, the three points $q^{k-2} \triangle e_{n-1} \triangle e_{n}, q^{k-1} \triangle e_{n-1} \triangle e_{n}, q^{k} \triangle e_{n-1} \triangle e_{n}$ have pairwise disjoint supports. In particular, for one of the three points, coordinates $n-1$ and $n$ are both set to 0 . Thus, for some $j \in\{k-2, k-1, k\}$,

$$
q_{n-1}^{j}=q_{n}^{j}=1 .
$$

Let $C$ be the member of cuboid $(S)$ corresponding to $q^{j}$. Then $C^{\text {odd }}=C_{j}^{\text {odd }} \cup\{2 n-3,2 n-1\}$ and $C^{\text {even }} \cap$ $\{2 n-2,2 n\}=\emptyset$. But then $B \cap C=\emptyset$, a contradiction.

Claim 2. There is a minimum weight cover $B$ of cuboid $(S)$, different from $\{1,2\}, \ldots,\{2 n-1,2 n\}$, such that $|B|=2$ and the two elements in $B$ have weight $\frac{\tau}{2}$.

Proof of Claim. Assume in the first case that there is a twisting of $S$ such that $w_{2 i} \geq \frac{\tau}{2} \geq w_{2 i-1}$ for each $i \in[n]$, and $\mathbf{0}$ is feasible. Let $C:=\{2 i: i \in[n]\}$ be the member of $\operatorname{cuboid}(S)$ corresponding to $\mathbf{0}$. By (c4), there exists a minimal cover $B$ such that $w(B) \leq \tau-2+|B \cap C|$. Clearly, $|B \cap C| \geq 2$, so

$$
\left|\left\{c \in[2 n]: w_{c} \geq \frac{\tau}{2}\right\}\right| \geq|B \cap C| \geq 2 .
$$

By (c5), $B$ has at most two elements of weight at least $\frac{\tau}{2}$, so $|B \cap C|=2$. However, by (c6), $B \subseteq C$ so $|B|=2$, and the two elements in $B$ have weight $\frac{\tau}{2}$, as required.

Assume in the remaining case that

$(\diamond)$ for every twisting of $S$ such that $w_{2 i} \geq \frac{\tau}{2} \geq w_{2 i-1}$ for each $i \in[n]$, the point $\mathbf{0}$ is infeasible.

Consider such a twisting. We may therefore apply Claim 1 . Let $p^{1}, \ldots, p^{k}$ be the feasible points of $S$ of minimal support, and let $C_{1}, \ldots, C_{k}$ be the corresponding members in $\operatorname{cuboid}(S)$. By Claim $1(1), k \geq 2$. We will show that $k=2$.

Suppose for a contradiction that $k \geq 3$. After a possible relabeling of $C_{1}, \ldots, C_{k}$, we may assume that

$(\star)$ if every element of $C_{j}^{\text {odd }}$ has weight $\frac{\tau}{2}$ for some $j \in[k]$, then every element of $C_{1}^{\text {odd }}$ has weight $\frac{\tau}{2}$.

By (c4), there is a minimal cover $B$ such that

$$
w(B) \leq \tau-2+\left|B \cap C_{1}\right|
$$

In particular, $\left|B \cap C_{1}\right| \geq 2$, implying in turn that $B$ does not contain any of $\{1,2\}, \ldots,\{2 n-1,2 n\}$, and $B^{\text {even }} \neq \emptyset$ by Remark 7.2. Thus, $B^{\text {even }}$ contains exactly one or two elements, by (c5).

Assume in the first case that $B^{\text {even }}$ contains exactly one element, say $2 n$. Then $2 n-1 \notin B$. By Claim 1 (2), we may assume that $B \cap C_{k}^{\text {odd }}=\emptyset$ and so $B \cap C_{k}=\{2 n\}$. The inequality above, together with Claim 1 (3), implies that

$$
w(B)=\tau-2+\left|B \cap C_{1}\right| .
$$


By Claim 1 (4), every element of $C_{k}^{\text {odd }}$ has weight $\frac{\tau}{2}$, so by $(\star)$, every element of $C_{1}^{\text {odd }}$ has weight $\frac{\tau}{2}$. By Claim 1 (2), $B \cap C_{i}^{\text {odd }} \neq \emptyset$ for each $i \in[k-1]$, and by Claim 1 (5), for each $i \in[k-1]$ and $c_{i} \in B \cap C_{i}^{\text {odd }}$,

$$
w_{c_{1}}+w_{c_{2}} \leq \sum_{i=1}^{k-1} w_{c_{i}}=\frac{\tau}{2}
$$

as $k \geq 3$. However, $w_{c_{1}}=\frac{\tau}{2}$ and $w_{c_{2}} \geq 1$ by (c2), a contradiction.

Assume in the remaining case that $B^{\text {even }}$ contains exactly two elements, say $2 n-2,2 n$. Then by (c6), $B \subseteq C_{1}$ and $w_{2 n-3}=w_{2 n-2}=w_{2 n-1}=w_{2 n}=\frac{\tau}{2}$. Since the twists of $S$ obtained after twisting either coordinates $n-1, n$ satisfy $(\diamond)$, it follows that both points $e_{n-1}, e_{n}$ are infeasible. It therefore follows from Claim 1 (6) that

$$
\left|\left\{j \in[k]: B \cap C_{j}^{\text {odd }}=\emptyset\right\}\right|=1 \text { or } 2 .
$$

On the other hand, as $C_{1}^{\text {odd }}, \ldots, C_{k}^{\text {odd }}$ are pairwise disjoint by Remark 7.2, and $B \subseteq C_{1}$, it follows that

$$
\left|\left\{j \in[k]: B \cap C_{j}^{\text {odd }}=\emptyset\right\}\right| \supseteq\{2, \ldots, k\} .
$$

Thus, $k \in\{2,3\}$. Since $k \geq 3$, it follows that $k=3$ and $B \cap C_{1}^{\text {odd }} \neq \emptyset$. Fix an element $c_{1} \in B \cap C_{1}^{\text {odd }}$, and for $j \in\{2,3\}$, pick an arbitrary element $c_{j} \in C_{j}^{\text {odd }}$. By Remark 7.2, $\left\{c_{1}, c_{2}, c_{3}\right\}$ is a minimal cover of cuboid $(S)$, so

$$
w_{c_{1}}+w_{c_{2}}+w_{c_{3}} \geq \tau
$$

However, $w_{c_{1}}=1$ by (c6), so

$$
w_{c_{2}}+w_{c_{3}} \geq \tau-1 \quad \forall c_{2} \in C_{2}^{\text {odd }}, \forall c_{3} \in C_{3}^{\text {odd }}
$$

As a result, for some $j \in\{2,3\}$, every element in $C_{j}^{\text {odd }}$ has weight at least $\left\lceil\frac{\tau-1}{2}\right\rceil=\frac{\tau}{2}$. Our twisting of $S$ implies that every element in $C_{j}^{\text {odd }}$ has weight exactly $\frac{\tau}{2}$. Thus, by $(\star)$, every element in $C_{1}^{\text {odd }}$ has weight $\frac{\tau}{2}$, so $1=w_{c_{1}}=\frac{\tau}{2}$, a contradiction to (c3).

As a result, $k=2$. For $i \in[2]$, pick $c_{i} \in C_{i}^{\text {odd }}$. By Remark 7.2, $\left\{c_{1}, c_{2}\right\}$ is a minimal cover. Moreover,

$$
\tau=\frac{\tau}{2}+\frac{\tau}{2} \geq w_{c_{1}}+w_{c_{2}} \geq \tau
$$

so $w_{c_{1}}=w_{c_{2}}=\frac{\tau}{2}$. As a result, $\left\{c_{1}, c_{2}\right\}$ is the desired minimal cover, thereby proving the claim.

After a possible twisting and relabeling, we may assume that $\{1,3\}$ is a minimal cover of $\operatorname{cuboid}(S)$ and $w_{1}=w_{3}=\frac{\tau}{2}$. In particular, the hypercube $\left\{x: x_{1}=x_{2}=0\right\}$ is infeasible. By the Plane Propagation Lemma, the two sets $S \cap\left\{x: x_{1}=1, x_{2}=0\right\}, S \cap\left\{x: x_{1}=0, x_{2}=1\right\}$ are hypercubes. Choose $I, J \subseteq\{3, \ldots, n\}$ and $I^{\prime}, J^{\prime} \subseteq\{3, \ldots, n\}$ such that

$$
S \cap\left\{x: x_{1}=1, x_{2}=0\right\}=\left\{x: x_{1}=1, x_{2}=0, x_{i}=1 \forall i \in I, x_{j}=0 \forall j \in J\right\}
$$

and

$$
S \cap\left\{x: x_{1}=0, x_{2}=1\right\}=\left\{x: x_{1}=0, x_{2}=1, x_{i}=1 \forall i \in I^{\prime}, x_{j}=0 \forall j \in J^{\prime}\right\} .
$$


Claim 3. The following statements hold:

(1) $w_{2 i-1} \geq \frac{\tau}{2}$ for each $i \in I \cup I^{\prime}$,

(2) $w_{2 j-1} \leq \frac{\tau}{2}$ for each $j \in J \cup J^{\prime}$,

(3) $w_{2 i-1}=\frac{\tau}{2}$ if $i \in I \cap J^{\prime}$ or $i \in I^{\prime} \cap J$,

(4) $I \cap I^{\prime}=\emptyset$ and $J \cap J^{\prime}=\emptyset$.

Proof of Claim. (1) For each $i \in I,\{3,2 i-1\}$ forms a cover, so $w_{2 i-1} \geq \tau-w_{3}=\frac{\tau}{2}$. For each $i \in I^{\prime}$, $\{1,2 i-1\}$ forms a cover, so $w_{2 i-1} \geq \tau-w_{1}=\frac{\tau}{2}$, so (1) follows. (2) For each $j \in J,\{3,2 j\}$ forms a cover, so $w_{2 j} \geq \tau-w_{3}=\frac{\tau}{2}$, so $w_{2 j-1} \leq \frac{\tau}{2}$. For each $j \in J^{\prime},\{1,2 j\}$ forms a cover, so $w_{2 j} \geq \tau-w_{1}=\frac{\tau}{2}$, so $w_{2 j-1} \leq \frac{\tau}{2}$, so (2) follows. (3) follows from (1) and (2). (4) Suppose for a contradiction that $I \cap I^{\prime} \neq \emptyset$ or $J \cap J^{\prime} \neq \emptyset$. Then for some element $c \in[2 n]-\{1,3\}$, the two sets $\{1, c\},\{3, c\}$ form minimal covers. However, $\{1,3\}$ is also a minimal cover, implying in turn that

$$
\operatorname{cuboid}(S) /([2 n]-\{1,3, c\}) \cong \Delta_{3},
$$

so cuboid $(S)$ is non-ideal by Remark 7.3, a contradiction to Corollary 1.5.

Claim 3 implies that there are feasible points $x^{1}, \ldots, x^{\tau}$ (repetition allowed) in the two hypercubes $S \cap\{x$ : $\left.x_{1}=1, x_{2}=0\right\}, S \cap\left\{x: x_{1}=0, x_{2}=1\right\}$ such that

$$
\left|\left\{j \in[\tau]: x_{i}^{j}=1\right\}\right|=w_{2 i-1} \quad \forall i \in[n] .
$$

Since $w_{2 i-1}+w_{2 i}=\tau$ for each $i \in[n]$, the members $C^{1}, \ldots, C^{\tau}$ of $\operatorname{cuboid}(S)$ corresponding to $x^{1}, \ldots, x^{\tau}$ yield a $w$-weighted packing, so $\nu(\operatorname{cuboid}(S), w) \geq \tau$, a contradiction.

\section{Concluding remarks}

We showed in Theorem 1.17 that $\left\{R_{k, 1}: k \geq 1\right\} \cup\left\{R_{5}\right\}$ are, up to isomorphism, the only strictly non-polar resistant sets that are half-dense. Question 1.16, asking for all of the resistant strictly non-polar sets, remains open. In fact, we cannot even answer the following question:

Question 8.1. Is there a non-polar resistant set $S \subseteq\{0,1\}^{n}$ such that $|S|<2^{n-1}$ ?

It seems to us that to answer Questions 1.16 and 8.1, we need to have a structure theorem for resistant sets. In two sequel papers [1,2], we provide structure theorems for natural classes of resistant sets - the structure theorems in turn answer Questions 1.16 and 8.1 for those classes.

Many of the theorems proved in this paper stemmed from propagations running in resistant sets. Do cubeideal sets in general have propagation features? The answer is yes; a weaker form of the Sight Propagation 


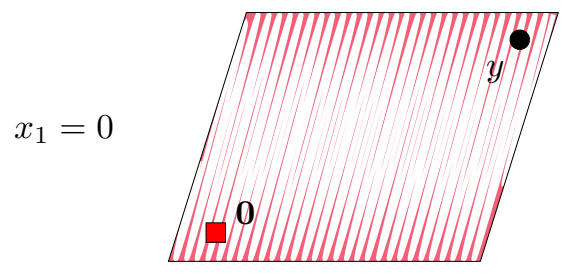

implies
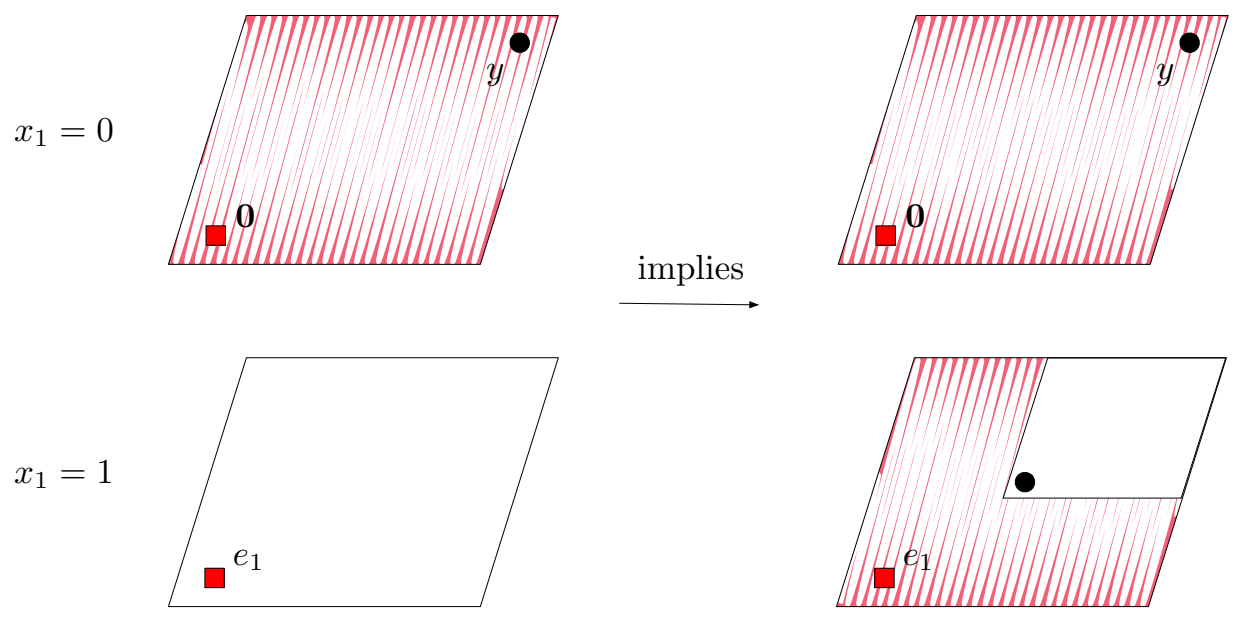

Figure 6: An illustration of Corollary 8.3, where $\left\{z \in S: z \leq y+e_{1}, z_{1}=1\right\}$ has a unique minimal point.

Lemma holds for cube-ideal sets. To elaborate, take an integer $n \geq 3$. A delta of dimension $n$ is the clutter over ground set $[n]$ whose members are

$$
\Delta_{n}:=\{\{1,2\},\{1,3\}, \ldots,\{1, n\},\{2,3, \ldots, n\}\} .
$$

$\Delta_{n}$ is a non-ideal clutter as $\left(\frac{n-2}{n-1} \frac{1}{n-1} \cdots \frac{1}{n-1}\right)$ is a fractional extreme point of the corresponding set covering polyhedron. As a result, ideal clutters do not have a delta minor by Remark 7.3. We will need the following tool for finding delta minors:

Theorem 8.2 ([4], Theorem 2.1). Let $\mathcal{C}$ be a clutter. If there are distinct members $C_{1}, C_{2}, C$ and an element $e$ such that $e \in C_{1} \cap C_{2}, e \notin C$ and $C_{1} \cup C_{2} \subseteq\{e\} \cup C$, then $\mathcal{C}$ has a delta minor through $e$.

As a consequence, we get the following weakening of Remark 4.7 for cube-ideal sets, which is illustrated in Figure 6.

Corollary 8.3. Take an integer $n \geq 1$ and a cube-ideal set $S \subseteq\{0,1\}^{n}$, where $\mathbf{0}, e_{1}$ are infeasible. Assume that $y$ is a minimal feasible point such that $y_{1}=0$. Then $\left\{z \in S: z \leq y+e_{1}, z_{1}=1\right\}$ has at most one minimal point.

Proof. Observe that every minimal point of $\left\{z \in S: z \leq y+e_{1}, z_{1}=1\right\}$, other than $y+e_{1}$, is also a minimal point of $S$. Suppose for a contradiction that $z^{1}, z^{2}$ are distinct minimal points of $\left\{z \in S: z \leq y+e_{1}, z_{1}=1\right\}$. Then $z^{1}, z^{2}$ must be different from $y+e_{1}$, so they are minimal points of $S$. Pick members $C, C_{1}, C_{2} \in \operatorname{ind}(S)$ such that $y=\chi_{C}, z^{1}=\chi_{C_{1}}, z^{2}=\chi_{C_{2}}$. Notice that $1 \in C_{1} \cap C_{2}, 1 \notin C$ and $C_{1} \cup C_{2} \subseteq\{1\} \cup C$. Theorem 8.2 implies that $\operatorname{ind}(S)$ has a delta minor, so $\operatorname{ind}(S)$ is non-ideal. Theorem 1.2 now applies and tells us that $S$ is not cube-ideal, a contradiction. 


\section{Acknowledgements}

This work was supported in parts by ONR grant 00014-18-12129, NSF grant CMMI-1560828, NSERC PDF grant 516584-2018 and IBS-R029-C1. We would like to thank two referees whose feedback improved the presentation of our paper.

\section{References}

[1] Abdi, A. and Cornuéjols, G.: Idealness and 2-resistant sets. Oper. Res. Lett. 47(5), 358-362 (2019)

[2] Abdi, A. and Cornuéjols, G.: The max-flow min-cut property and \pm 1 -resistant sets. Submitted.

[3] Abdi, A., Cornuéjols, G., Guričanová, N., Lee, D.: Cuboids, a class of clutters. J. Combin. Theory Ser. B (2019), https://doi.org/10.1016/j.jctb.2019.10.002.

[4] Abdi, A., Cornuéjols, G., Pashkovich, K.: Ideal clutters that do not pack. Math. Oper. Res. 43(2), 533-553 (2018)

[5] Abdi, A., Feldmann, A.E., Guenin, B., Könemann, J., Sanità, L.: Lehman's theorem and the directed Steiner tree problem. SIAM J. Discrete Math. 30(1), 141-153 (2016)

[6] Abdi, A. and Pashkovich, K.: Delta minors, delta free clutters, and entanglement. SIAM J. Discrete Math. 32(3), 1750-1774 (2018)

[7] Angulo, G., Ahmed, S., Dey, S., Kaibel, V.: Forbidden vertices. Math. Oper. Res. 40(2), 350-360 (2014)

[8] Berge, C.: Balanced matrices. Math. Program. 2(1), 19-31 (1972)

[9] Conforti, M. and Cornuéjols, G.: Clutters that pack and the max-flow min-cut property: a conjecture. The Fourth Bellairs Workshop on Combinatorial Optimization (1993)

[10] Conforti, M., Cornuéjols, G., Zambelli, G.: Integer programming. Springer (2014)

[11] Cornuéjols, G.: Combinatorial optimization, packing and covering. SIAM, Philadelphia (2001)

[12] Cornuéjols, G., Guenin, B., Margot, F.: The packing property. Math. Program. Ser. A 89(1), 113-126 (2000)

[13] Cornuéjols, G. and Novick, B.: Ideal 0,1 matrices. J. Combin. Theory Ser. B 60, 145-157 (1994)

[14] Edmonds, J.: Optimum branchings. J. Res. Nat. Bur. Standards 71B(4), 233-240 (1967)

[15] Edmonds, J. and Fulkerson, D.R.: Bottleneck extrema. J. Combin. Theory Ser. B 8, 299-306 (1970)

[16] Edmonds, J. and Giles, R.: A min-max relation for submodular functions on graphs. Annals of Discrete Math. 1, 185-204 (1977) 
[17] Edmonds, J. and Johnson, E.L.: Matchings, Euler tours and the Chinese postman problem. Math. Prog. 5, 88-124. (1973)

[18] Fulkerson, D.R.: Blocking and anti-blocking pairs of polyhedra. Math. Program. 1, 168-194 (1971)

[19] Guenin, B.: A characterization of weakly bipartite graphs. J. Combin. Theory Ser. B 83, 112-168 (2001)

[20] Guenin, B.: Perfect and ideal 0, \pm 1 matrices. Math. Oper. Res. 23(2), 322-338 (1998)

[21] Hoffman, A.J.: A generalization of max flow-min cut. Math. Prog. 6(1), 352-359 (1974)

[22] Hoffman, A.J. and Kruskal J.B.: Integral boundary points of convex polyhedra. In Linear inequalities and related systems (eds. Kuhn H.W. and Tucker A.W.). Ann. Math. Studies 38, 223-246 (1956)

[23] Kőnig, D.: Graphs and matrices (in Hungarian). Matematikai és Fizikai Lapok 38:116-119 (1931)

[24] Lee, J.: Cropped cubes. J. Combin. Optimization 7(2), 169-178. (2003)

[25] Lehman, A.: The width-length inequality and degenerate projective planes. DIMACS Vol. 1, 101-105 (1990)

[26] Lovász, L.: Minimax theorems for hypergraphs. Lecture Notes in Mathematics 411, Springer-Verlag 111$126(1972)$

[27] Nobili, P. and Sassano, A.: (0, \pm 1$)$ ideal matrices. Math. Program. 80(3), 265-281 (1998)

[28] Seymour, P.D.: The matroids with the max-flow min-cut property. J. Combin. Theory Ser. B 23, 189-222 (1977) 